\title{
Alpha-ketoglutaric acid ameliorates hyperglycemia in diabetes by inhibiting hepatic gluconeogenesis via serpina1e signaling
}

Gang Shu ( $\sim$ shugang@scau.edu.cn )

South China Agricultural University https://orcid.org/0000-0002-1321-4396

\section{Yexian Yuan}

South China Agricultural University

Jia Sun

Zhujiang Hospital

Canjun Zhu

South China Agricultural University

Jinlong Feng

South China Agricultural University

\section{Zewei Ma}

South China Agricultural University

\section{Wentong Peng}

South China Agricultural University

\section{Cong Yin}

South China Agricultural University

\section{Guli Xu}

South China Agricultural University

\section{Pingwen $\mathrm{Xu}$}

University of Illinois at Chicago

\section{Yuwei Jiang}

University of Illinois at Chicago

\section{Songbo Wang}

South China Agricultural University

\section{Xiaotong Zhu}

South China Agricultural University

\section{Ping Gao}

South China Agricultural University

\section{Lina Wang}

South China Agricultural University

\section{Qianyun Xi}


South China Agricultural University

\section{Yongliang Zhang}

South China Agricultural University

\section{Qingyan Jiang}

South China Agricultural University https://orcid.org/0000-0003-3466-2443

\section{Article}

Keywords: AKG, Diabetes, Serpina1e, Hepatic Gluconeogenesis

Posted Date: November 20th, 2020

DOI: https://doi.org/10.21203/rs.3.rs-104995/v1

License: (c) (1) This work is licensed under a Creative Commons Attribution 4.0 International License. Read Full License 
4 Yexian Yuan ${ }^{1,5}$, Jia Sun ${ }^{2,5}$, Canjun Zhu ${ }^{1}$, Jinlong Feng ${ }^{1}$, Zewei Ma ${ }^{1}$, Wentong Peng ${ }^{1}$, Cong Yin ${ }^{1}$, Guli

$5 \mathrm{Xu}^{1}$, Pingwen $\mathrm{Xu}^{3}$, Yuwei Jiang ${ }^{4}$, Songbo Wang ${ }^{1}$, Lina Wang ${ }^{1}$, Xiaotong Zhu ${ }^{1}$, Ping Gao ${ }^{1}$, Qianyun $\mathrm{Xi}^{1}$,

$6 \quad$ Yongliang Zhang ${ }^{1}$, Qingyan Jiang ${ }^{1}$ and Gang Shu ${ }^{1, *, \dagger}$

$7 \quad{ }^{1}$ Guangdong Laboratory of Lingnan Modern Agriculture and Guangdong Province Key Laboratory of

\section{Alpha-ketoglutaric acid ameliorates hyperglycemia in diabetes by inhibiting hepatic}

\section{gluconeogenesis via serpina1e signaling}

\section{Guangdong Laboratory of Lingnan Modern Agriculture and Guangdong Province Key Laboratory of}

Animal Nutritional Regulation, National Engineering Research Center for Breeding Swine Industry,

College of Animal Science, South China Agricultural University, 483 Wushan Road, Tianhe District, Guangzhou, Guangdong 510642, China

${ }^{2}$ Zhujiang Hospital, Southern Medical University, 510280, China

${ }^{3}$ Division of Endocrinology, Department of Medicine, The University of Illinois at Chicago, Chicago, Illinois, 60612, USA

${ }^{4}$ Department of Physiology and Biophysics, The University of Illinois at Chicago, Chicago, Illinois, 60612, USA

${ }^{5}$ Co-first author

${ }^{\dagger}$ Lead contact

*Correspondence should be addressed to:

Gang Shu (Lead contact)

483 Wushan Road, Tianhe District, Guangzhou, Guangdong 510642, China

E-mail: shugang@scau.edu.cn

Telephone: $+86-20-85284901$

Fax: $+86-20-85284901$ 
25 The authors have declared that no conflict of interest exists.

26

27

28

29

30

31

32

33

34

35

36

37

38

39

40

41

42

43

44

45 
Summary: While resistance exercise effectively improves overall health in diabetic patients, the

47 underlying biological mechanism by which resistance exercise improves metabolic function and glucose homeostasis remain mostly unknown. Previously, we identified a myometabolite-mediated metabolic pathway that is essential for the beneficial effects of resistance exercise on metabolic function. We found that resistance exercise-induced $\alpha$-ketoglutaric acid (AKG) stimulates muscle hypertrophy and fat loss through 2-oxoglutarate receptor 1 (OXGR1)-dependent adrenal activation. Here, we provided evidence for the beneficial effects of AKG on glucose homeostasis in a diet-induced obesity (DIO) mouse model, which are independent of OXGR1. We showed that circulating AKG levels are negatively correlated with the fraction of blood glycated hemoglobin (HbA1c) in both humans and mice and significantly decreased in DIO mice. Consistently, pharmacological elevation of AKG effectively decreased body weight, blood glucose, and hepatic gluconeogenesis without changing insulin sensitivity and glucose tolerance in DIO mice. Notably, OXGR1KO blocked the inhibitory effects of AKG on body weight but failed to affect AKG's suppression on blood glucose and hepatic gluconeogenesis, indicating distinct mechanisms for AKG's regulation on energy balance and glucose homeostasis. In supporting this view, we showed that serpinale, a member of protease inhibitor serpins superfamily, mediates the direct inhibitory effects of AKG on gluconeogenesis in both in vitro hepatocytes and liver slice. By using a liver-specific serpinale deletion mouse model, we further demonstrated that liver serpinale is required for the inhibitory effects of AKG on hepatic gluconeogenesis and hyperglycemia in DIO mice. Finally, we provided in vitro evidence to support a model in which AKG decreases hepatic gluconeogenesis by targeting trimethylation of lysine 27 on histone 3 (H3K27me3) in seprinale promoter region. Our studies established an important role of AKG 
67 in glucose homeostasis, and identified the AKG-serpina1e pathway as potential therapeutic targets to

68

69

70

71

72

73

74

75

76

77

78

79

80

81

82

83

84

85

86

87

88

89

90

91

92

93

94

95

96

97

98

99

100

101

102

103

104

105

106

107

80

83

85

89

0

2

3

5

6

.

2$$
06
$$

07 attenuate hyperglycemia.

Keywords: AKG/Diabetes/Serpina1e/Hepatic Gluconeogenesis.

(1)

15

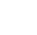

1

84

87

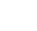

103


Type 2 diabetes mellitus (T2DM) is a growing global health problem, which decreases life spans through premature morbidity or mortality from associated metabolic diseases ${ }^{1}$. Exercise has long been established as an important non-pharmacological therapeutic strategy for the management of T2DM. Combined with diet control and behavior intervention, exercise effectively reduces the risk of diabetes ${ }^{2}$. Previous studies have shown that exercise improves glucose homeostasis partially through exerciseinduced myobolites (or myometabolites). Several exercise-induced myobolites have been identified to exert different beneficial effects on glucose balance. Specifically, leucine promotes insulin secretion ${ }^{3}$, lactate acts as a substrate for gluconeogenesis ${ }^{4}$, and succinate robustly improves glucose tolerance ${ }^{5}$, all of which elicit complex responses involved in glucose metabolism ${ }^{6}$. These myometabolites are potential therapeutic targets for T2DM, which actively prevents glycemic-related diseases by acting as exercise mimetics. While metabolite therapies for T2DM are emerging, metabolite-induced beneficial effects on glucose homeostasis still face a major obstacle of low long-term therapeutic efficiency. Here regular physical exercise. biological processes, including intestinal innate immunity ${ }^{8}$, antioxidative defense ${ }^{9}$, energy production

Alpha-ketoglutaric acid (AKG), a citric acid cycle intermediate also known as 2-oxoglutarate, is a serum metabolic signature of acute resistance exercise ${ }^{7}$. It has been shown to plays a vital role in a variety of ${ }^{10}$, epigenetic modification ${ }^{11}$, and tumor suppression ${ }^{12}$. Interestingly, we recently found that $2 \%$ AKG water supplementation effectively prevents diet-induce obesity (DIO) by increasing brown adipose tissue temperature, oxygen consumption, and whole-body metabolism in a 2-oxoglutarate receptor 1 (OXGR1)-dependent mechanism ${ }^{13,14}$. Notably, data from high-fat-diet (HFD)-fed middle-aged mice 
highlight that this keto-acid also contributes to glycemic control by acting as a nutrient to improve glucose tolerance and insulin sensitivity ${ }^{15}$. In line with this observation, water supplementation of $1 \%$ AKG alleviates hyperglycemia by increasing whole-body insulin sensitivity in DIO rats ${ }^{16}$. These studies consistently demonstrated benefic effects of AKG on metabolic balance and glucose homeostasis. Here we aim to further define the role of AKG on glycemic control in humans and different diabetic mouse models, and further identify the biological molecular mechanism of these actions.

In this study, we found that serum AKG is negatively correlated with human plasma glycated hemoglobin A1c (HbA1c), the standard diabetic management biomarker for diabetic patients ${ }^{17}$. Consistently, in DIO, chemical-induced type I diabetes (T1D), and $d b / d b$ mouse models, AKG supplementation significantly decreased the activity of liver gluconeogenesis enzyme flux and ameliorated hyperglycemia. Using in vitro hepatocyte and liver slice culture models, we showed that AKG directly suppresses hepatic gluconeogenesis by inhibiting rate-limiting enzymes. We subsequently identified serpinale (serine or cysteine peptidase inhibitor, clade A, member 1e) as a transcriptomic signature induced by $\mathrm{AKG}$ treatment in the liver. We further indicated that $\mathrm{AKG}$ promotes serpinale expression by decreasing the trimethylation of lysine 27 on histone 3 (H3K27me3) in seprinale promoter region. Finally, by using a liver-specific seprinale deletion mouse model, we established the key role of serpinale in AKG-induced suppression on hepatic gluconeogenesis and hyperglycemia in DIO mice. Overall, our studies established a vital role of AKG in glucose homeostasis and identified the AKG-serpinale pathway as potential therapeutic targets to mitigate T2DM. 


\section{Results}

152

153

AKG is negatively correlated with blood glucose.

Previous studies showed that AKG is increased by resistance exercise and negatively correlated with human body mass index (BMI) ${ }^{14,18}$. Here, we found that plasma AKG concentrations in male mice (a population including chow-fed C57BL/6 mice, DIO C57BL/6 mice, and $d b / d b$ mice) exhibited a statistically significant inverse relationship with blood hemoglobin A1c $(\mathrm{HbA1c}, \mathrm{R}=-0.84, \mathrm{P}<0.0001$, Fig. 1A). To further distinguish pre-diabetic obese and non-diabetic obese mice, HbA1c $\geq 6.5 \%$ was used as a criteria for the diagnosis of pre-diabetes ${ }^{19}$. We found the plasma AKG levels in non-diabetic obese mice are significantly higher than levels in pre-diabetic obese mice (Fig. 1B), suggesting a potential role of AKG in blood glucose control. Notably, we showed that AKG concentration exhibited a similar inverse relationship with HbA1c levels in human plasma $(\mathrm{R}=-0.39, \mathrm{P}<0.001$, Fig. 1C). Conversely, the concentrations of AKG-related metabolites, including glutamine $(\mathrm{Glu}, \mathrm{R}=0.23, \mathrm{P}=0.09)$, alphaketoisovaleric acid ( $\alpha$ keval, $\mathrm{R}=0.33, \mathrm{P}<0.05$ ), succinic acid (SUA, $\mathrm{R}=0.35, \mathrm{P}<0.05$ ), $\alpha$-ketoleucine (akehex, $\mathrm{R}=0.47, \mathrm{P}<0.01$ ), and fumaric acid (FUMA, $\mathrm{R}=0.47, \mathrm{P}<0.01$ ), showed a positive relationship with HbA1c levels in human plasma. Collectively, blood AKG is negatively associated with glycemia, suggesting a physiological role in glycemic control.

\section{AKG treatment improves glucose homeostasis.}

The negative correlation between circulating AKG levels and glycemia prompted us to investigate the role of AKG in glycemic control. Specially, ad libitum chow-fed or HFD-fed male C57BL/6 mice were provided with drinking water supplemented with $2 \%$ AKG. This dose of AKG supplementation has been shown to increase circulating AKG level up to a dose comparable to that observed in mice 
receiving resistance exercise, suggesting a physiological boost of circulating $A K G{ }^{7}$. While AKG supplementation significantly decreased blood glucose and HbAlc levels in HFD-fed mice, it failed to affect blood glucose in chow-fed mice (Fig. 2A-C), suggesting diet-dependent glycemia-lowering effects of AKG. In HFD-fed mice, AKG also decreased body weight gain and fat mass while increased lean mass without changing food intake (Fig. S1A-C), which is consistent with our previous observations ${ }^{14}$. One possibility is that anti-obesity effects of AKG lead to the enhanced insulin receptor sensitivity and further improve glucose homeostasis. However, we found that AKG increased circulating insulin levels and improved pyruvate tolerance without changing glucose tolerance, insulin sensitivity, or blood glucagon levels (Fig. 2D-H), suggesting that reduced obesity may not be the main contributor for AKG-induced anti-hyperglycemia effects in DIO mice. AKG-induced glycemialowering effects are unlikely explained by an improvement in insulin response and instead argued in favor of a possible contribution by hepatic gluconeogenesis.

In support of this view, AKG treatment dramatically decreased the mRNA expression of gluconeogenesis rate-limiting enzymes, including PEP carboxykinas (PEPCK), glucose 6-phosphatase (G6Pase), and fructose 1,6-bisphosphatas (FBP) in the liver (Fig. 2I). Consistently, AKG also decreased hepatic enzyme activity of PEPCK, G6Pase, and FBP (Fig. 2J - L). Liver plays an essential role in the control of glucose homeostasis by regulating various pathways of glucose metabolism, including glycogenesis, glycolysis, and gluconeogenesis. The enzymes involved in these pathways are required for the proper functioning of glycemic control ${ }^{20}$. Our data showed that AKG supplementation inhibited the glycolysis pathway as indicated by decreased mRNA expression of phosphofructokinase (Pfkl), aldolase fructose-bisphosphate A (Aldoa), enolase 1 (Eno1) and lactate dehydrogenase A in the liver 
195

196

197

(Ldha, Fig. S1D). Besides, AKG also suppressed the pentose phosphate pathway (PPP) and glycogenesis pathway as showed by downregulated mRNA levels of glycerate kinase (Glyctk), fructose-bisphosphatase 1 (FBP1), phosphodiesterase 1 (Enpp1), glycogen synthase 2 (Gys2) and acylCoA dehydrogenase (Acadm), as well as by upregulated the mRNA levels of fructose-bisphosphate C aldolase (Aldoc, Fig. S1D). While AKG supplementation increased the storage of glucose (glycolysis, pentose phosphate, and glycogenesis), it also reduced the source of glucose (gluconeogenesis), which ultimately leads to lower blood glucose levels. Together, our data suggest that AKG may prevent DIOinduced hyperglycemia by inhibiting hepatic gluconeogenesis and stimulating insulin secretion.

\section{Glycemia-lowering effect of AKG is mediated by suppression of hepatic gluconeogenesis.}

We next examined if acute AKG treatment produces similar glycemia-lowering effects as we observed after chronic AKG supplementation. Specifically, male C57BL/6 mice were intraperitoneal (i.p.) injected with $\mathrm{AKG}$ at a dose of $10 \mathrm{mg} / \mathrm{kg}$. This dose of $\mathrm{AKG}$ treatment has been shown to increase circulating $\mathrm{AKG}$ concentration up to a comparable level observed after chronic $2 \%$ AKG water supplementation ${ }^{7}$. The result showed that AKG-treated mice exhibited lower blood glucose at $0.5,1,2$, and 3 hours (hrs) after injection compared with saline-treated mice (Fig. 3A). Blood HbA1c content was not changed at $3 \mathrm{hrs}$ after i.p. injection of AKG (Fig. 3B). Notably, AKG administration also increased plasma insulin levels (Fig. 3C) and decreased hepatic enzyme activities of PEPCK, G6Pase, and FBP (Fig. 3D-F). These results indicate that like chronic supplementation, acute i.p. injection of AKG also inhibits hepatic gluconeogenesis and stimulates insulin secretion, further supporting that AKG's anti-hyperglycemia effects are independent of its anti-obesity effects. 
217 Consistent with our observations that both chronic and acute AKG treatment increased insulin release

218

219

220

221

222

224

(Fig. 2F and 3C), AKG has been recently found to act as a metabolic signaling molecule to regulate pancreatic $\beta$-cells function and promote insulin secretion ${ }^{21}$. It is possible that $\mathrm{AKG}$-induced insulin indirectly acts on the liver to reduce hepatic gluconeogenesis. To investigate whether AKG directly inhibits hepatic gluconeogenesis or indirectly by promoting insulin secretion, we tested AKG's effects in a chemical-induced type I diabetes (T1D) mouse model. Specifically, impairment of islet function and insulin secretion was induced by 7-day i.p injection of streptozotocin (STZ) in male C57BL/6 mice.

Compared to intact mice (insulin level $\sim 0.5 \mathrm{ng} / \mathrm{mL}$, Fig.3C), STZ-treated mice exhibit significantly lower serum insulin levels $(0.2 \sim 0.5 \mathrm{pg} / \mathrm{mL})$, which were not changed by AKG treatment (Fig. S2C). These findings suggested impaired insulin secretion and verified our T1D mouse model. We found that impaired insulin secretion did not affect the regulatory effects of AKG on glucose homeostasis, as indicated by decreased blood glucose levels (Fig. S2A), unchanged blood HbA1c content (Fig. S2B), and decreased hepatic enzyme activities of PEPCK, G6Pase, and FBP (Fig. S2D-F). These results indicate that $\mathrm{AKG}$ inhibits hepatic gluconeogenesis in an insulin-independent mechanism.

To test AKG's effects in other diabetic models, we i.p. injected AKG in $d b / d b$ mice, which is the most widely used mouse model for diabetes ${ }^{22,23}$. Consistent with the anti-hyperglycemia effects in DIO and TZD-treated mice, acute AKG treatment significantly decreased blood glucose (Fig. S2G) and hepatic gluconeogenesis enzyme activities (Fig. S2J-L), as well as increased serum insulin levels without affected the HbA1c content in $d b / d b$ mice (Fig. S2H-I). Collectively, results from both acute and chronic AKG treatment support a notion that AKG prevents hyperglycemia by suppressing hepatic gluconeogenesis in an insulin-independent mechanism in diabetic mice. 


\section{AKG directly inhibits hepatic gluconeogenesis in an OXGR1-independent mechanism}

As an important signal molecule in organism, AKG regulates physiological progress via acting on related target organs, such as adipose tissue, intestinal, brain, and muscle ${ }^{24}$. We next asked whether decreased hepatic gluconeogenesis induced by AKG results from a direct effect on the liver. We examined the effects of AKG treatment in in vitro primary hepatocyte or ex vivo liver slice culture model.

To induce a insulin resistance hepatic cell model, primary hepatocyte obtained from chow-fed male C57BL/6 mice were treated with $0.25 \mathrm{mM}$ palmitic acid (PA) for $24 \mathrm{hrs}{ }^{25}$. We observed that while 100 $\mu \mathrm{M}$ AKG treatment failed to decrease the enzyme activities of PEPCK, G6Pase, and FBP in control primary hepatocyte without PA treatment (Fig. 4A-D), it significantly decreased the activities of these enzymes in primary hepatocyte with PA treatment (Fig. 4E-G). These data suggest a direct effect of AKG in in vitro primary hepatocyte. In line with these observations, we also found decreased enzyme activities of PEPCK, G6Pase, and FBP in PA-treated liver slices (Fig. 4H-K) and primary hepatocytes derived from DIO mice (Fig. 4L-O). Thus, the results from both in vitro and ex vivo hepatic models showed that AKG directly acts on the liver to suppress gluconeogenesis.

OXGR1 has been identified as the primary mediating receptor for anti-obesity effects of AKG ${ }^{7,26}$. Interestingly, while OXGR1 deletion blocked the inhibitory effects of chronic AKG supplementation on body weight and fat mass (Fig. 5A-B), it failed to affect AKG's effects on blood glucose, blood HbA1c level, hepatic activity of PEPCK, G6Pase and FBP, and pyruvate tolerance (Fig. 5C-H). These results suggest that OXGR1 is not required for glycemia-lowering effects of AKG.

\section{Serpina1e is required for the suppressive effects of AKG on hepatic gluconeogenesis.}


To explore the mechanism of AKG-induced gluconeogenesis suppression, we investigated the

262

263

264

265

266

267

268

269

270

271 transcriptomic alteration induced by AKG treatment in the liver of DIO mice. We found that multiple genes showed profound transcriptional changes (Fig. 6A-B). These genes included serpinale, encoding a protein called $\alpha 1$-antitrypsinhighly, which is a type of serine protease inhibitor ${ }^{27}$; selenium binding protein 2 (selenbp2), which had been identified as the major target for acetaminophen in the liver ${ }^{28}$; cytochrome P450, family 2, subfamily c, polypeptide 70 (cyp2c70), which regulates the hydroxylated muricholic acids formation ${ }^{29}$; and cytochrome P450, family 4, subfamily a, polypeptide $12 \mathrm{~b}$ (cyp4a12b), which conducts $\omega$-hydroxylation of fatty acids ${ }^{30}$. All these genes are highly expressed in the liver and involved in liver metabolism. To further test whether these genes are essential for AKG-induced suppression on hepatic gluconeogenesis, we generated in vitro loss-of-function primary hepatocyte models by using siRNA to target serpinale, selenbp2, cyp2c70, or cyp4a12b, respectively. We found that the siRNA-treated primary hepatocyte showed significantly lower mRNA expression of targeted genes compared with control scrambled siRNA-treated cells (Fig. 6E, S3A, S3E, S3I), thereby validating primary hepatocyte knockdown models. We showed that the knockdown of serpinale, but not selenbp2, cyp2c70, and cyp4a12b, effectively abolished the inhibitory effects of AKG on the activities of PEPCK, G6Pase, and FBP in PA-treated primary hepatocyte (Fig. S3B-D, F-H, J-L, Fig. 6F-H), suggesting a vital role of serpinale in AKG-induced hepatic gluconeogenesis. In supporting this view, we found that the mRNA expression of hepatic serpinale in DIO or $d b / d b$ mice is lower than that in chow-fed mice (Fig. 6C), suggesting the metabolic relevance of hepatic serpinale. Consistently, we also showed that chronic AKG supplementation increased the phosphorylation of focal adhesion kinase (FAK, Fig. 6D), a tyrosine-phosphorylated protein mediating the regulatory effects of serine protease inhibitors on cell physiological metabolism. In summary, our results suggest an essential role of 
serpinale in AKG-induced inhibition on hepatic gluconeogenesis

To further determine the role of serpinale in the systemic effects of AKG on glucose homeostasis, we generated a serpinale liver-specific KO mouse model (Alb-serpina1 $\mathrm{e}^{-/}$). Specifically, Alb-Cre mice were crossed with LSL-Cas9-EGFP mice to generate Alb-Cre/LSL-Cas9-EGFP (Alb-Cas9), a mouse model with Cas9 selectively overexpressed in Alb positive liver cells. Subsequently, an adenoassociated virus harboring single-guide RNAs (sgRNAs) targeting serpinale or scramble sgRNAs was intravenously injected to generate serpinale-deficient mice or control mice, respectively. We found that the Alb-serpina1 $\mathrm{e}^{-/-}$mice showed absent serpina1e mRNA expression in the liver compared with the control mice (Fig. 6I), which validates our $\mathrm{KO}$ model. Consistent with the in vitro primary hepatocyte model, liver-specific serpina1e KO abolished the AKG-induced decreases of blood glucose (Fig. 6J $\mathrm{K})$ and inhibition on hepatic gluconeogenesis as indicated by the activities of PEPCK, G6Pase and FBP (Fig. 6N-P). Conversely, liver-specific serpina1e KO failed to disrupt the effects of acute AKG treatment on plasma HbA1c content and insulin secretion (Fig. 6L-M). These findings support a model that $\mathrm{AKG}$ acts on hepatic serpinale signaling to reduce gluconeogenesis and glycemia.

\section{The inhibitory effects of AKG on hepatic gluconeogenesis rely on JMJD3-H3K27me3 pathway.}

AKG regulates metabolic processes mainly through the membrane receptor (OXGR1), sensors, or epigenetic modification ${ }^{31-33}$. Serving as a vital substrate and cofactor for epigenetic modifications including RNA methylation and histone methylation, AKG plays a key role in beige adipose thermogenesis, macrophage orchestration, and mitochondrial glucose metabolism ${ }^{15,33,34}$. It is possible that AKG inhibits hepatic gluconeogenesis through epigenetic mechanisms. We first investigated 
305

306

307

308

309

310

311

312

AKG's effects on RNA m6A modification (N6-methyladenosine), which is the most prevalent RNA modification and has been reported in numerous human diseases, including several cancers ${ }^{35,36}$. In primary hepatocyte cell, we tested the AKG's effects on the mRNA expression of three major types of enzymes involved in m6A methylation: writers, erasers, and readers ${ }^{35,37,38}$. We found AKG failed to affect the mRNA expression of these writers, readers, and erasers (Fig. S4A-B), suggesting an alternative epigenetic mechanism.

Subsequently, we further tested AKG's effects on histone demethylase, which has been reported as an epigenetic drug target for metabolic disease, such as obesity, hepatosteatosis, and type 2 diabetes ${ }^{39,40}$. We specifically detected the mRNA expression of Jumonji domain-containing protein-3 (JMJD3), lysine specific demethylase 1 (LSD1), and lysine demethylase 6A (UTX), the most widely known histone demethylases. We found that AKG increased the mRNA expression of JMJD3 without affecting LSD1 and UTX in primary hepatocyte cells (Fig. 7A). JMJD3 is one of the primary demethylases of histone H3 lysine 27 trimethylation (H3K27me3), a repressive epigenetic mark that prevents gene expression ${ }^{41}$. We wondered whether AKG inhibits hepatic gluconeogenesis by promoting JMJD3dependent demethylation of H3K27 on the promoters of serpinale. To test it, we generated JMJD3knockdown cell lines by transfecting primary hepatocyte cells with plasmid harboring siRNA targeting JMJD3 (Fig. 7B). JMJD3 knockdown abolished inhibitory effects of AKG on H3K27me3 levels and enzyme activities of PEPCK, G6Pase and FBP (Fig. 7C-F). Collectively, these results support a mediating role of JMJD3-H3K27me3/Serpina1e signaling in the inhibitory effects of AKG on hepatic gluconeogenesis in vitro. 
327 Histone epigenetic modification occurs in nucleus. To investigate how cytoplasmic AKG gets into cell

328

329

\section{6} nucleus to regulate serpina1e-H3K27me3, we further analyzed the transcriptomic changes of dicarboxylic acid transporters in the liver of AKG-treated male DIO mice. We found that AKG supplementation significantly increased the mRNA expression of solute carrier family 25 member 11 (SLC25A11) and sodium-dependent dicarboxylate cotransporter member 2 (SLC13A2, Fig. 7G), the primary carrier involved in citrate reabsorption ${ }^{42}$. Notable, AKG failed to affect the mRNA expression of others potential carrier for AKG, including solute carrier family 25 member 10 (SLC25A10), solute carrier family 25 member 20 (SLC25A20), solute carrier family 25 member 21 (SLC25A21), solute carrier family 25 member 1 (SLC25A1), solute carrier family 13 member 3 (SLC13A3), and solute carrier family 13 member 5 (SLC13A5). Based on these observations, we speculated that dicarboxylate transporters SLC25A11 and SLC13A2 may participate in the transport of AKG into the nucleus. To test this view, we generated SLC25A11- or SLC13A2-knockdown cell lines by transfecting primary hepatocyte cells with plasmid harboring siRNA targeting SLC25A11 or SLC13A2, respectively (Fig. 7H and S4C). We found that SLC25A11 but not SLC13A2 knockdown abolished AKG-induced hepatic gluconeogenesis suppression (Fig. 7I-K, S4D-F). Consistently, AKG specifically induced cytoplasmto-nucleus translocation of SLC25A11 in primary hepatocyte cell (Fig. 7L), identifying SLC25A11 as the primary transporter for AKG.

(1)

5

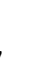

(1) 
The major finding of our study is that AKG, an exercise-induced myobolite ${ }^{14,18}$, impairs hepatic gluconeogenesis and improves glucose homeostasis in diabetic mice. Our study revealed a negative correlation between $\mathrm{AKG}$ and glucose in the blood of both humans and mice. Long-term systemic treatment of AKG effectively lowered blood glucose levels, which were associated with decreased hepatic gluconeogenesis and increased insulin secretion, in DIO and $d b / d b$ mice. Notably, the inhibitory effects of AKG on glycemia and hepatic gluconeogenesis were preserved in TZD-treated T1D and OXGR1KO mice, indicating an insulin- and OXGR1-independent mechanism. We also find acute administration of AKG induced similar glucose responses as observed in mice chronically treated with

AKG. Finally, we provided in vitro, ex vivo, and in vivo evidence to support that AKG directly acts on JMJD3/H3K27me3/serpina1e pathway to inhibit hepatic gluconeogenesis and decrease blood glucose. Collectively, we provided a novel mechanism by which AKG inhibits hepatic gluconeogenesis and ameliorates hyperglycemia in diabetic mice. 
371

372

is possible that the glycemia-lowering effect of AKG is a secondary response to decreased adiposity. In this study, we provided multiple lines of evidence to support a distinct mechanism for AKG's effects on glucose homeostasis. First, we found plasma AKG levels in non-diabetic obese mice are significantly higher than diabetic obese mice. This evidence suggests that the association between AKG and blood glucose is independent of body weight. Second, acute AKG treatment produce similar glycemialowering effects as observed in DIO and $d b / d b$ mice chronically treated with AKG. Finally, our previous observations found that AKG reduces obesity in an OXGR1-dependent mechanism ${ }^{14,15}$. However, OXGR1KO did not affect AKG's inhibitory effects on glycemia and hepatic gluconeogenesis in DIO mice, suggesting an OXGR1-independent mechanism. Collectively, AKG-induced glycemia-lowering effect is independent of its anti-obesity effect.

One interesting glucose phenotype is that both acute and chronic AKG treatments increase blood insulin but not glucagon levels in DIO and $d b / d b$ mice. These findings are consistent with previous reports that AKG stimulates insulin secretion through hypoxia-inducible factor-prolyl hydroxylases (PHDs) in clonal $\beta$-cells as well as rodent and human islets ${ }^{21,43}$. Glucose-induced secretion of insulin is wellknown to inhibit hepatic glucose production ${ }^{44}$, suggesting a possibility that AKG increases insulin secretion to indirectly inhibit hepatic gluconeogenesis. However, evidence from two separate mouse models implies an alternative mechanism. Specifically, depletion of endogenous insulin by STZ treatment did not affect AKG's effects on blood glucose and hepatic gluconeogenesis, suggesting an insulin-independent mechanism. In line with these observations, in liver-specific serpinale KO mice, while the stimulatory effects on blood insulin levels persisted, the inhibitory effects of AKG on blood glucose and hepatic gluconeogenesis were abolished. Therefore, the glycemia-lowering effect of AKG 
does not rely on insulin secretion.

Glucose homeostasis is a dynamic process maintained through glucose consumption in metabolic tissues and production in the liver ${ }^{45}$. In mammals, liver is the most important organ to regulate body glucose metabolism through glycolysis, pentose phosphate pathway, and gluconeogenesis. Hepatic gluconeogenesis is an essential therapeutic target for T2DM, and several commercial available drugs, including metformin ${ }^{46}$ and pioglitazone ${ }^{47}$, effectively improve glucose homeostasis by acting on gluconeogenesis. Here, we found that chronic AKG treatment effectively inhibited mRNA expression of genes involved in glycolysis, pentose phosphate, and glycogen metabolism in the liver of DIO mice, suggesting the reduced glucose utilization. Moreover, AKG supplementation significantly suppressed the mRNA expression of gluconeogenesis gene, suggesting the increased glucose source. Consistently, chronic AKG treatment also improved pyruvate tolerance and inhibited the activities of rate-limiting enzymes for gluconeogenesis. Like chronic treatment, acute AKG administration also suppressed essential gluconeogenesis enzyme activities in in vivo DIO, T1D and $d b / d b$ mouse models as well as in vitro primary hepatocyte and ex vivo liver slice. Collectively, AKG generate a robust direct inhibition on hepatic gluconeogenesis.

To explore the potential mechanisms for AKG's effects on hepatic gluconeogenesis, we screened transcriptomic changes induced by AKG treatment in the livers of DIO mice. We identified serpinale, a member of serine protease inhibitors (serpins), as an AKG-induced transcriptomic signature. Serpins are the largest and most broadly distributed superfamily of protease inhibitors and Serpinal is highly expressed in the liver ${ }^{27,48}$. Importantly, FAK, serpinale's downstream effector, has also been shown to regulate human liver disease ${ }^{49}$. It is possible that serpinale mediates the inhibitory effects of AKG on 
hepatic gluconeogenesis and blood glucose. To directly test it, we generated a serpinale-knockdown

417 primary hepatocyte model and liver-specific serpinale-KO mouse model. In supporting our hypothesis, serpina1e knockdown or KO effectively abolished AKG's effects on hepatic gluconeogenesis. These results reveal a potential novel role of $\mathrm{AKG} /$ serpina1 signaling in hepatic gluconeogenesis regulation.

Emerging evidence demonstrates that AKG serves a signal molecule integrating metabolism and aging process by reducing epigenetic age in both humans and animals. Specifically, AKG regulates the function of essential enzymes that influence epigenetic modifications to modulate gene expression and intracellular metabolic process. These enzymes include 2-oxoglutarate-dependent demethylase (FTO), which is involved in RNA demethylation, and the Jumonji domain-containing proteins (JMJD) family, which is the major histone demethylases ${ }^{50,51}$. The epigenetic modifications induced by these two enzymes have been linked to metabolic disease. For example, N6-methyladenosine (m6A) methylation, one of the most common RNA modifications, plays a vital role in cancer therapy ${ }^{52}$, obesity, and glucose 
SLC25A11 were up-regulated in response to AKG supplementation. The $\mathrm{Na}(+) /$ dicarboxylate cotransporter SLC13A2 (also known as NaDC-1) was mainly reabsorbed succinate and citrate ${ }^{56}$. In response to glucose stimulate, SLC13A2 could result in obviously secretion of insulin, which promotes us to consider its role on glucose regulation ${ }^{57}$. The malate- $\alpha$-ketoglutarate antiporter SLC25A11 (also known as oxoglutarate carrier, OGC), which exists in the mitochondrial inner membrane, is responsible for the malate-asparate shuttle ${ }^{58}$. Most of the transport $(>80 \%)$ in liver or kidney tissue could be accounted for the oxoglutarate carrier (OGC, SLC25A11), which mediate electroneutral exchange of dicarboxylates for 2-oxoglutarate ${ }^{59}$. As the important shuttle molecule, SLC25A11 play a role in glucose homeostasis, such as insulin secretion ${ }^{60}$, gluconeogenesis regulation ${ }^{61}$. Here, we found that loss function of SLC25A11 effectively abolished AKG induced gluconeogenesis decrease. However, SLC13A2 interference could not reverse the inhibitory effect of gluconeogenesis by AKG. At the same time, we found that AKG treatment promoted the translocation of SLC25A11 in primary hepatocyte from cytoplasm to nucleus. This evidence suggests that cytoplasm-to-nucleus transport of AKG is probably mediated through SLC25A11.

In conclusion, we found that systemic supplementation of AKG prevent diabetic elevation of blood glucose by inhibiting hepatic gluconeogenesis in a serpinale-H3K27me3 dependent mechanism. 


\section{Animals}

Mice were housed in a temperature/humidity-controlled environment $\left(23{ }^{\circ} \mathrm{C} \pm 3{ }^{\circ} \mathrm{C} / 70 \% \pm 10 \%\right)$ on a

12-hr light/12-hr dark cycle (6 am and $6 \mathrm{pm})$. C57BL/6 mice in this paper were purchased from the stated, the mice were maintained ad libitum on standard mouse chow (protein $18.0 \%$, fat $4.5 \%$, and carbohydrate 58\%, Guangdong Medical Science Experiment Center, Guangzhou, Guangdong, China) and drinking water. All groups in one experiment contained an individual mouse with the same strain and sex. C57BL/6 mice were used for long-term or acute experiments to investigate the effects of AKG on blood glucose. The liver-specific serpina1e KO mice (Alb-cre crossed with cas9 mice, then injected with serpinale-sgRNA HBAAV) were generated on a C57BL/6 background. They were used to investigate the effects of short-term AKG administration. Care of all animals and procedures in South China and were approved by the Animal Subjects Committee of South China Agricultural University.

\section{Primary hepatocyte preparation}

The primary hepatocyte fraction was obtained from 10 weeks C57BL/6 male mice as described previously ${ }^{62,63}$. Mice were anesthetized by isoflurane and then cut vertically until the liver, portal vein, and inferior vena cava were sufficiently exposed. The flow rate was increased to $7-9 \mathrm{~mL} /$ minute with

HBSS. The entire volume of HBSS was perfused through the liver. When the reservoir was just about to run out of HBSS, $70 \mathrm{~mL}$ of the digestion medium was poured. Type IV collagenase $(100 \mathrm{U} / \mathrm{ml}$; 
490

491

492

493

494

495

496

497

498

499

500

501

502

503

504

505

506

507

508

509

510

511

512

17104019, Thermo Fisher) was used for digestion, and the flow rate was about $8 \mathrm{~mL} / \mathrm{min}$. Dulbecco's Modified Eagle's Medium (DMEM) with $25 \mathrm{mM}$ glucose and 10\% FBS was used for isolation and plating, and viability as determined by trypan blue staining was $>90 \%$ for all preparations. Cells were plated on collagen-coated $\left(8 \mu \mathrm{g} / \mathrm{cm}^{2}\right)$ plates. After the cells attached, they were washed once with DMEM-low and culture media (with 10\% FBS) was added again for 3-4 hours. Plating was conducted for the first 4-5 hours. Cells were kept in a serum-free medium containing 5 to $25 \mathrm{mM}$ glucose overnight, and all cells were used within $30 \mathrm{~h}$ of plating.

\section{Primary tissue culture of liver}

Mouse liver tissue slice obtained from 12-week-old C57BL/6 mice were cultured in high-glucose $\operatorname{DMEM}\left(11965175\right.$, Thermo Fisher Scientific, Carlsbad, CA, USA) at $37^{\circ} \mathrm{C}$ in a humidified atmosphere that contained $5 \% \mathrm{CO}_{2}$. The high-glucose DMEM was supplemented with $10 \%$ fetal bovine serum (FBS) (16000044, Thermo Fisher Scientific), $100 \mathrm{mg} / \mathrm{L}$ of streptomycin sulfate (11860038, Thermo Fisher Scientific), and 100000 units/L of penicillin sodium. The liver was sliced to about $200 \mu \mathrm{m}$ by a vibration slicer (VF-300 Microtome, U.S) and then cultured for further treatment.

\section{Association between plasma AKG level and blood glucose in Chinese adults}

This observational study was conducted in Huadong Sanatorium (Wuxi, China) and Zhujiang hospital (Guangzhou, China) between 2018 and 2019. Forty-two Chinese volunteers (36 males and 6 females) aged 26 to 85 were recruited from Huadong Sanatorium (Wuxi, China) and Zhujiang hospital (Guangzhou, China). All volunteers were required to complete a self-assessment form one week before sample collection, including age, gender, and symptoms of other diseases. Volunteers with other diseases that affect blood glucose level and diabetes treatment history were excluded. Each volunteer's 
blood samples were collected and stored in EDTA tubes. Plasma samples were obtained by centrifugation $\left(4{ }^{\circ} \mathrm{C}, 4000 \times \mathrm{g}\right.$ for $\left.20 \mathrm{~min}\right)$ and used for spectrophotometry detection of heparin and glucose. The whole test procedure was reviewed and approved by the Human Subjects Ethics Committee of Huadong Sanatorium, and written consent was obtained from each volunteer. The plasma AKG levels were measured by LC-MS/MS analysis (UPLC 1290-6470A QQQ liquid chromatographymass spectrometry instrument, Agilent Technologies).

\section{RNA interference in primary hepatocyte}

RNA interference was conducted as we previously described ${ }^{14}$. The serpinale siRNA and negative control (NC) siRNA were purchased from GenePharma Co., Ltd. (Shanghai, China) and transfected into primary hepatocyte by using lipofectamine reagents (Invitrogen, Carlsbad, CA, USA) according to the manufacturer's instructions. The sequences of siRNA targeting serpinale are 5'UGGCUCAUGCCUGAUGCUATT-3' (sense) and 5'-UAGCAUCAGGCAUGAGCCATT-3' (antisense). The sequences of siRNA targeting selenbp2 are 5'-CCGACGAGCAAAUCUCAUUTT-3' (sense) and 5'-AAUGAGAUUUGCUCGUCGGTT-3' (anti-sense). The sequences of siRNA targeting 5'-GGGCUUAUUCUGAUGUCAUTT-3' (sense) and 5'-

AUGACAUCAGAAUAAGCCCTT-3' (anti-sense). The sequences of siRNA targeting cyp2c70 are 5'CCAAGGGCACAAGUGUAAUTT-3' (sense) and 5'-AUUACACUUGUGCCCUUGGTT -3' (antisense). The sequences of siRNA targeting SLC25A11 are 5'-GCCACUUCUGCGCCAGCAUTT-3' (sense) and 5'-AUGCUGGCGCAGAAGUGGCTT-3' (anti-sense). The sequences of siRNA targeting 
sense). The sequences of NC siRNA are 5'-UUCUCCGAACGUGUCACGUTT-3' (sense) and 5'ACGUGACACGUUCGGAGAATT-3' (anti-sense). An aliquot of transfected cells was collected to determine serpinale gene expression. Another aliquot of transfected cells was treated with 0 or $100 \mu \mathrm{M}$ AKG for intracellular experiments.

\section{Liver specific-serpina1e KO mouse model}

AAV virus vector carrying paired single-guide RNAs (AAV-sgRNAs-Serpinale) targeting serpinale (sgRNA binding sites with $20.1 \mathrm{~kb}$ interval were located in exon 1-5 of the serpinale gene) was generated and packed by Cyagen (Suzhou, China). The serpinale sgRNA-A1: ACACAGAGGCCACTCTATTGA, sgRNA-A2: CAGCACAGAGGTCCCTCATAT. Alb-Cre mice were crossed with LSL-Cas9-EGFP mice to generate Alb-Cre/LSL-Cas9-EGFP (Alb-Cas9), a mouse model with Cas9 selectively overexpressed in Alb positive liver cells. For six-week-old mice, male AlbCas9 mice were i.p injected with AAV-sgRNAs-serpina1e $\left(1 \times 10^{12} \mathrm{GC} / \mathrm{ml}\right)$ to generate a liver-specific sepina1e deletion mouse model (Alb-serpina1 $\mathrm{e}^{-/-}$). At 10 weeks of age, male Alb-serpina1 $\mathrm{e}^{-/-}$and control mice (LSL-Cas9-EGFP mice injected with AAV-sgRNAs-serpina1e) were i.p injected with saline or $10 \mathrm{mg} / \mathrm{kg}$ AKG. Blood glucose was measured at 0.5, 1, 2, 3, 4, 5, and $6 \mathrm{~h}$. At the end of the experiment, serum was collected and centrifuged at $20000 \mathrm{~g}$ and $4{ }^{\circ} \mathrm{C}$ for $20 \mathrm{~min}$. The HbA1c, insulin, and liver gluconeogenesis enzymes were tested after the mice were euthanized.

\section{Western blot analysis}

Western blot analysis was performed as described previously ${ }^{64}$. Total protein lysates $(20 \mu \mathrm{g})$ were immunoblotted with rabbit-anti-p-FAK (1:1000, \#3283, CST), rabbit-anti-FAK (1:1000, \#13009, CST), 
rabbit-anti- $\beta$-Tubulin (1:50000, AP0064, Bioworld Technology, Inc., St. Louis Park, MN, USA), followed by donkey-anti-goat HRP conjugated secondary antibody or goat-anti-rabbit HRP conjugated secondary antibody (1:50000, bs-0294D or bs-0295G, Bioss, Woburn, MA, USA). The levels of tubulin served as the loading control.

\section{Relative quantitative PCR analysis}

Real-time PCR (RT-PCR) assay was conducted as described previously ${ }^{64}$. Total mRNA was extracted by a HiPure RNA Kit (R4130-02, Magen, China) and digested with DNase I. The total mRNA (1 $\mu \mathrm{g})$ was reverse-transcribed to cDNA by oligo (dT) 18 primer. Then, SYBR Green relative quantitative RTPCR was performed according to published protocols ${ }^{65}$. Results were normalized by the expression of housekeeping gene $\beta$-actin.

\section{Absolute quantitative PCR analysis}

Absolute quantitative RT-PCR assay was performed according to previous papers ${ }^{14,66}$. The cDNA samples from mouse liver tissues were first generated as described in relative quantitative RT-PCR analyses. The $\mathrm{Ct}$ value of each gene was obtained for further analysis. The specific PCR amplification product was purified by electrophoresis and gel extraction by using an agarose gel recovery kit (D211102, Magen BioSciences, Waltham, MA, USA) to generate a standard curve for serpinale gene. The DNA concentration of each product was measured by NanoDrop (2000c, Thermo Fisher Scientific).

The absolute copy number of each sample was calculated according to the following formula: $\mathrm{C}=\mathrm{A} / \mathrm{B}$ $\times 6.02 \times 10^{14}$, where A is the concentration obtained by OD260 analysis $(\mathrm{ng} / \mu \mathrm{L}), \mathrm{B}$ is the molecular weight of the synthesized DNA (Daltons), and C is the copy number of the synthesized DNA 
(copies $/ \mu \mathrm{L}$ ). Subsequently, eight-fold serial dilution was carried out on each purified PCR product 12 times. The dilutions of each product were used as the templates for SYBR Green quantitative real-time PCR to target the gene by using an ample amount of the above-mentioned primers. The standard curve of each gene was plotted as a linear regression of the $\mathrm{Ct}$ values versus the log of the copy number.

\section{Immunofluorescence staining}

Primary hepatocyte cells were cultured in 6-well plates with an adhesive coverslip. At about $50 \%$ coverage of coverslip, the cell was treated with 0 or $100 \mu \mathrm{M} A K G$ for $6 \mathrm{~h}$ and incubated with the primary rabbit-anti-SLC25A11 antibody (1:1000, ab80464, abcam) at a temperature of $4{ }^{\circ} \mathrm{C}$ overnight, followed by goat-anti-rabbit FITC conjugated secondary antibody (1:1000, bs-0295G, Bioss) for $1 \mathrm{~h}$. The primary hepatocyte cells were mounted on slides and coverslipped with mounting medium with DAPI (H-1200, Vector Laboratories, Burlington, ON, Canada). Fluorescence images were obtained using Nikon Eclipse Ti-s microscopy (Nikon Instruments, Tokyo, Japan).

\section{ChIP and real-time PCR}

Chromatin immunoprecipitation (ChIP) assays were performed with a ChIP kit (LOT3432949, Millipore) and performed according to certain protocols ${ }^{33,67,68}$. Briefly, primary hepatocyte were fixed with $1 \%$ formaldehyde for 15 min and then lysed with lysis buffer. To shear the DNA, cell lysates were sonicated and the extracts were clarified by centrifugation method. After preclearing with protein Gagarose beads, antibodies were added and incubated at $4{ }^{\circ} \mathrm{C}$ overnight on a $360^{\circ}$ rotator. Then, protein G-agarose beads were added, rotated for $1 \mathrm{~h}$ at $4{ }^{\circ} \mathrm{C}$ to collect immunoprecipitated complexes. The samples were washed once with low-salt buffer, once with high-salt buffer, once with LiCl buffer, and 
602

603

604

605

606

607

608

609

610

611

612

613

614

615

616

617

618

619

620

621

622

623

then twice with Tris-EDTA buffer, and they were finally eluted with elution buffer. After reversing cross links between protein and DNA by heating at $65^{\circ} \mathrm{C}$ for $4 \mathrm{~h}$, the DNA was purified and subjected to RTPCR analysis. DNA (total chromatin) was input as the endogenous control. The primers that were used to amplify the serpinale promoter are forward 5'- GGGTGTTCTAACTGCTTTCT $-3^{\prime}$ and reverse 5'-

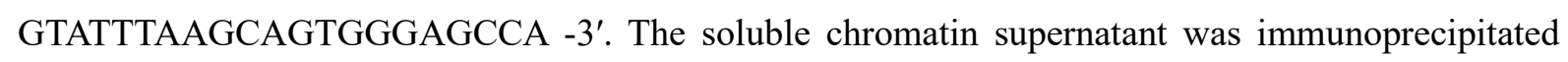
with anti-H3K27me3 (Millipore 07-449). Immunoprecipitated DNA and input DNA were analyzed by using q-RT-PCR, and the results are presented as the percentage of input.

\section{Transcriptomics}

Transcriptomic was performed as described before ${ }^{14}$. Samples from HFD C57BL/6 male mice liver tissue were used for transcriptomic signature analysis. Untargeted transcriptomics profiling was conducted on the Illumina platform (Novogene, Beijing, China) by Novogene Co., Ltd (Beijing, China).

The liver tissue sample preparation procedures can be referred to the previously published protocol with minor revisions ${ }^{69}$. First, RNA was extracted by a HiPure RNA Kit (R4130-02, Magen, China) and digested with DNase I. RNA integrity was assessed by the RNA Nano 6000 Assay Kit of the Bioanalyzer 2100 system (Agilent Technologies, CA, USA). Total RNA ( $3 \mu \mathrm{g}$ per sample) was used for RNA sample preparations. Next, RNA sequencing libraries were generated using NEBNext ${ }^{\circledR}$ UltraTM RNA Library Prep Kit for Illumina ${ }^{\circledR}$ (NEB, USA) following the manufacturer's recommendations, and index codes were added to attribute sequences to each sample. Finally, further analysis was conducted.

\section{UPLC-Orbitrap-MS/MS analysis for metabolites}

The methods were performed as described previously ${ }^{14}$. One hundred $\mu \mathrm{L}$ of serum was transferred to a 
$1.5 \mathrm{~mL}$ EP microtube with $500 \mu \mathrm{L}$ of methanol (mass spectrometry grade) and then added to each sample to fully remove protein. Serum metabolite content was analyzed using LC-MS/MS analysis (UPLC 1290-6470A QQQ liquid chromatography-mass spectrometry instrument, Agilent Technologies).

\section{AKG effects on STZ-induced T1D}

Eight-week-old male C57BL6/J mice were i.p injected with 60 mg/kg STZ (S0130, Sigma, MO, USA) once a day for 7 days. One week later, the blood glucose levels were measured and the mice with blood glucose levels $>450 \mathrm{mg} / \mathrm{dL}$ were considered diabetic; thus, they were chosen for the following studies. Mice were randomly divided into two groups according to body weight, age, and blood glucose. The selected mice received i.p injections of AKG $(10 \mathrm{mg} / \mathrm{kg})$ or saline. The blood glucose levels of the mice were recorded for $6 \mathrm{~h}$.

\section{Effect of AKG on reducing blood glucose in $\mathrm{db} / \mathrm{db}$ mice}

Ten-week-old male $\mathrm{db} / \mathrm{db}$ mice were i.p injected with $10 \mathrm{mg} / \mathrm{kg} \mathrm{AKG}$ or saline. Blood glucose was measured at $0,1,2,3,4,5$, and $6 \mathrm{~h}$. At the end of the experiment, serum HbAlc, insulin level and liver PEPCK, G6Pase, and FBP enzyme activity were examined.

\section{IPGTT, PTT, and ITT}

Intraperitoneal glucose tolerance test (IPGTT) and PTT were performed after overnight fasting. An injection of $1 \mathrm{~g} / \mathrm{kg}$ (body weight) of glucose was given to the mice, and blood glucose levels were measured subsequently at different time points. An injection of $1 \mathrm{~g} / \mathrm{kg}$ (body weight) of pyruvate was given to the mice, and blood glucose levels were measured subsequently at different time points. ITT was performed after $4 \mathrm{~h}$ of fasting. The mice were i.p injected with a single dose of insulin $(1 \mathrm{U} / \mathrm{kg})$, after which the blood glucose levels were measured.

\section{Serum insulin, HbA1c level, and liver enzyme activity assay}


650

651

652

653

654

655

656

657

658

659

660

661

662

663

664

665

666

667

668

669

670

671

and $\mathrm{HbA1c}$ were measured using ELISA (MM-0159M2, Jiangsu Meimian Industrial Co., LTD). The enzyme activity of PEP carboxykinase (PEPCK), fructose 1,6-bisphosphatase (FBP), and glucose 6phosphatase (G6Pase) were measured using commercially available kits according to the manufacturer's instructions (Solarbio, China).

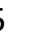

6

7

Serum levels of insulin were measured using ELISA (CSB-E05071m, CUSABIO BIOTECH CO., LTD)

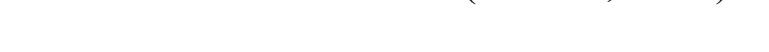

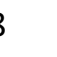

9

0

61

62

63

64

\section{5}

\section{6}

67


673 Statistical analyses were performed using GraphPad Prism 7.0 statistics software (Chicago, IL, USA).

674 Statistical analyses methods were chosen based on the design of each experiment and indicated in the

675 figure legends. The data were presented as mean \pm SEM. $\mathrm{P} \leq 0.05$ was considered statistically significant.

676

677

678

679

680

681

682

683

684

685

686

687

688

689

690

691

692

693

694

695

696

697

698

699

700

701

702

703

704

705

706 
707

708

709

710

711

712

713

714

715

716

717

718

719

720

721

722

723

724

725

726

727

728

729

730

731

\section{4}

\section{5}

\section{Author Contributions}

Y. Y. and J. S. are the main contributors in the conduct of the study, data collection and analysis, data interpretation, and manuscript writing. C. Z., Z. M., J. F., W. P., C. Y., G. X., P. X., and Y. J. contributed to the conduct of the study. S. W., L. W., X. Z., P. G., Q. X., Q. J, and Y. Z. contributed to the manuscript writing and data interpretation. G. S. contributed to the study design, data interpretation, and manuscript writing.

4

15

16

17

8

9

20

\section{1}

2

23


733 This work was supported by grants from National Natural Science Foundation of China (31790411 to

734 Q. J.), The Local Innovative and Research Teams Project of Guangdong Province (2019BT02N630 to 735 G.S.), National Key Point Research and Invention Program (2016YFD0501205 to G.S and 7362018 YFD0500403 to G. S.), National Natural Science Foundation of China (31572480 to G.S.), 737 Research and development projects in key areas of Guangdong Province (2019B020218001 to G.S.), 738 Innovation Team Project in Universities of Guangdong Province (2017KCXTD002 to G. S.), National 739 Institute of Diabetes and Digestive and Kidney Diseases from National Institutes of Health 740 (R00DK107008 to P. X., K01DK111771 to Y. J.).

741 
Figure 1. Serum AKG level is negatively with HbA1c.
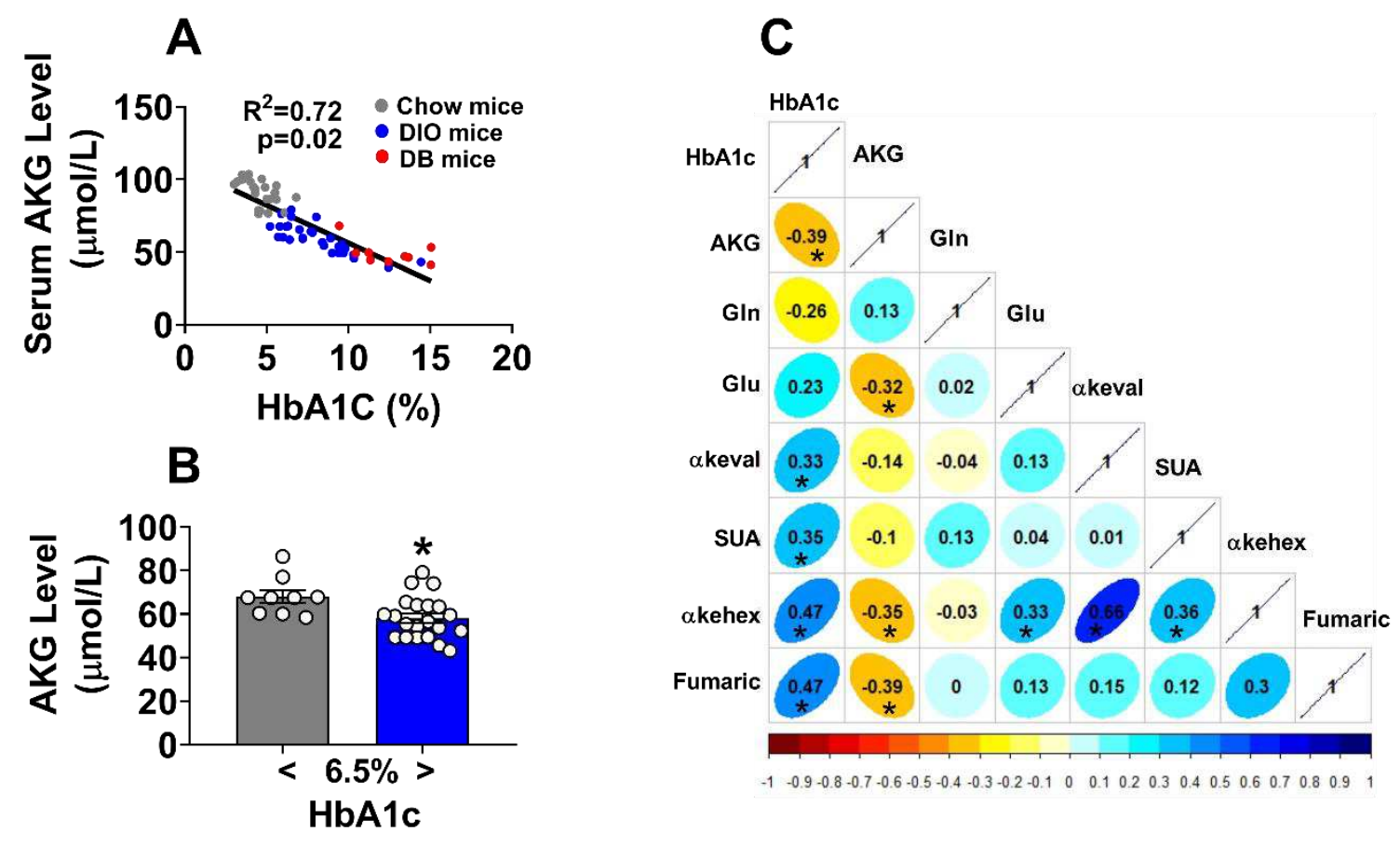

744

(A). Two-tailed Pearson's correlation coefficient analysis of plasma AKG level and HbA1c in mice. Chow male mice were fed a chow diet at 8 weeks of age $(n=21)$. For diet induced obesity mice (DIO), eight-weekold C57BL/6 male mice were fed HFD for 12 weeks $(\mathrm{n}=30)$. DB $(\mathrm{db} / \mathrm{db}$ diabetes $)$ male mice were fed a chow diet at 10 weeks of age $(n=9)$.

(B). Plasma AKG level and HbA1c in DIO mice. Eight-week-old C57BL/6 male mice were fed HFD for 12 weeks ( $\mathrm{n}=9-21$ per group).

(C). Two-tailed Pearson's correlation coefficient analysis of plasma AKG and related metabolite level with blood glucose in Chinese adults (36 males and 6 females). (Gln: glutamine; Glu: glutamic acid; $\alpha$-keval: alpha-ketoisovaleric acid; SUA: succinic acid; $\alpha$-kehex: $\alpha$-ketoleucine; FUMA: fumaric acid; AKG: oxoglutaric acid). 
A

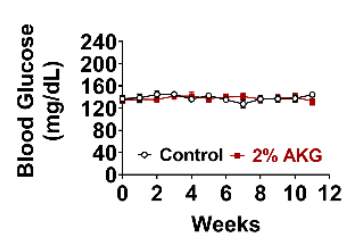

$\mathbf{E}$
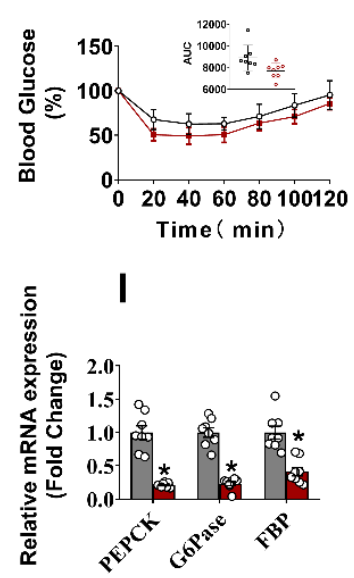

B

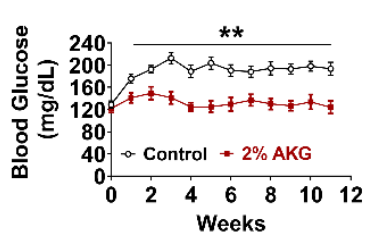

F

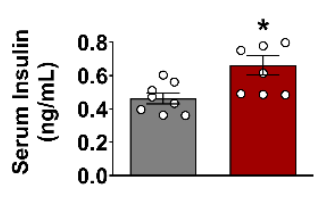

$\mathbf{J}$

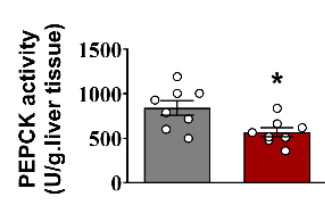

C

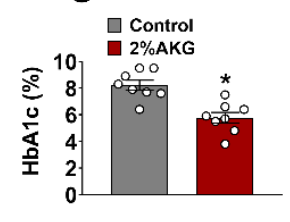

G

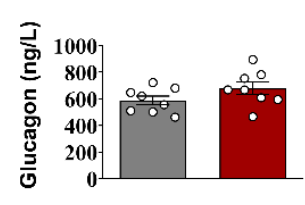

$\mathbf{K}$

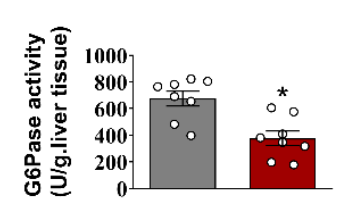

D

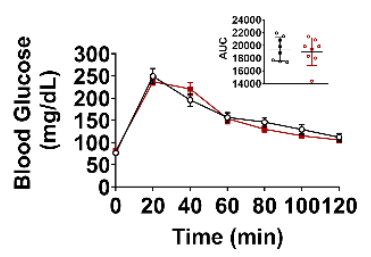

H

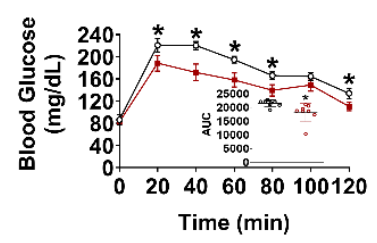

$\mathbf{L}$

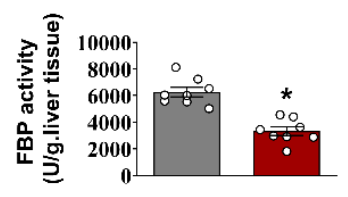

764

(A). Blood glucose of male C57BL/6 mice. At 8 weeks of age, mice were fed a chow diet and received tap water or water supplemented with $2 \%$ AKG for 11 weeks ( $n=8$ per group).

(B-C). Blood glucose (B) and serum HbAlc level (C) of male C57BL/6 mice. At 12 weeks of age, mice were switched to HFD and received tap water or water supplemented with $2 \%$ AKG for 11 weeks ( $n=8$ per group). (D-E). Glucose tolerance test $(1 \mathrm{~g} / \mathrm{kg})(\mathrm{D})$ and insulin tolerance test $(1 \mathrm{U} / \mathrm{kg})(\mathrm{E})$ in male C57BL/6 mice. At 12 weeks of age, mice were switched to HFD and received tap water or water supplemented with $2 \%$ AKG for 11 weeks ( $\mathrm{n}=8$ per group). switched to HFD and received tap water or water supplemented with $2 \%$ AKG for 11 weeks ( $n=8$ per group). (H). Pyruvate tolerance test (PTT, $1 \mathrm{~g} / \mathrm{kg}$ ). At 12 weeks of age, mice were switched to HFD and received tap water or water supplemented with $2 \%$ AKG for 11 weeks ( $n=8$ per group). 

8 per group). $(\mathrm{n}=8$ per group)

Data information: Results are presented as mean \pm SEM. In (B), $(\mathrm{H}) . * \mathrm{p} \leq 0.05$ by two-way ANOVA followed by post-hoc Bonferroni tests. In (C), (F), and (J-L), *p $\leq 0.05$ by non-paired Student's t-test.

801 

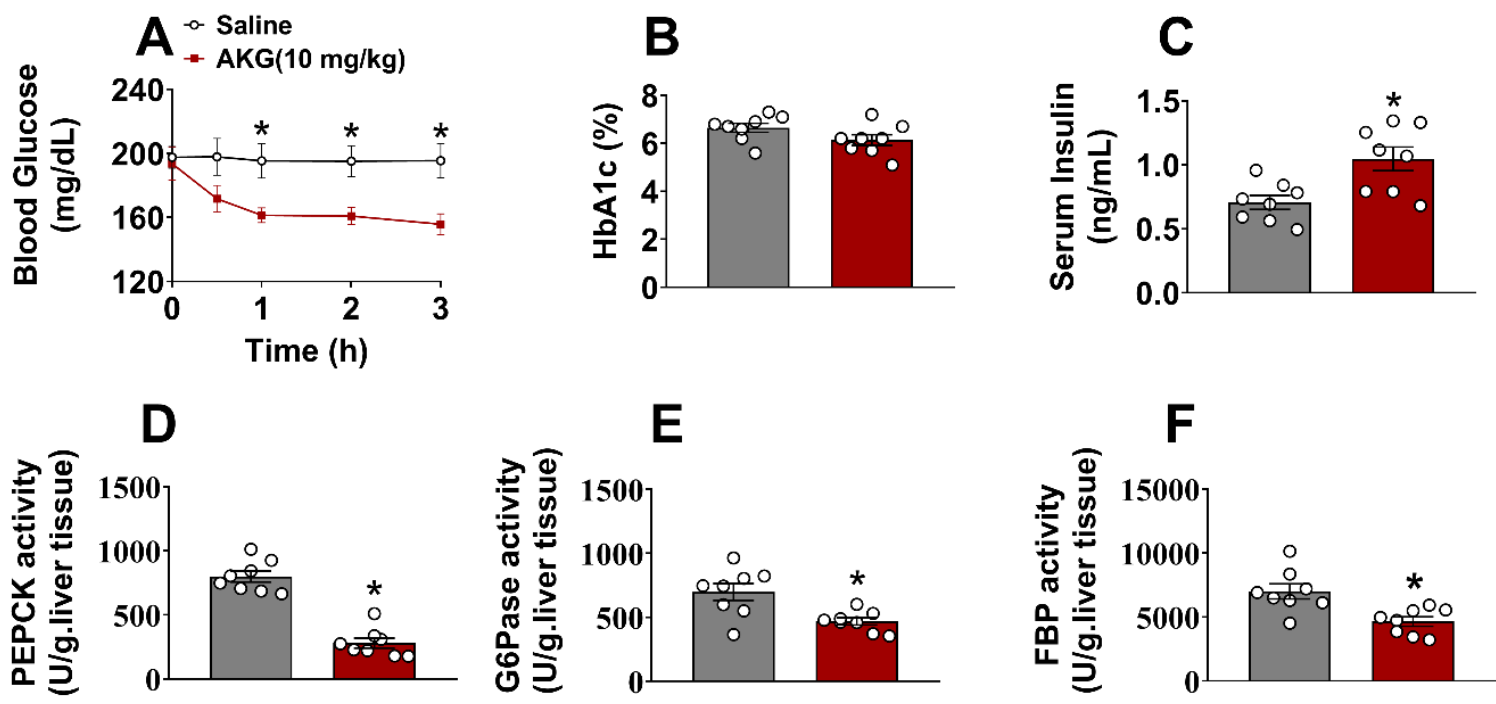

806

807

(A). Blood glucose concentration-time profile obtained from male C57BL/6 mice (10 weeks old) fed with HFD i.p saline or AKG (10 mg/kg body weight). The blood glucose was tested at $0,0.5,1,2$, and $3 \mathrm{hrs}$ after injection ( $\mathrm{n}=8$ per group).

810 (B-C). Serum HbA1c level (B) and insulin level (C) in male C57BL/6 mice (10 weeks) fed with HFD i.p saline or AKG $(10 \mathrm{mg} / \mathrm{kg})$ for $3 \mathrm{hrs}$. (D-F). The activity of PEPCK (D), G6Pase (E), and FBP (F) in liver of male C57BL/6 mice (10 weeks) fed with HFD i.p saline or AKG $(10 \mathrm{mg} / \mathrm{kg})$ for $3 \mathrm{hrs}$. Data information: Results are presented as mean \pm SEM. In (A), $* \mathrm{p} \leq 0.05$ by two-way ANOVA followed by post-hoc Bonferroni tests. In (C-F), ${ }^{*} \mathrm{p} \leq 0.05$ by non-paired Student's t-test. 


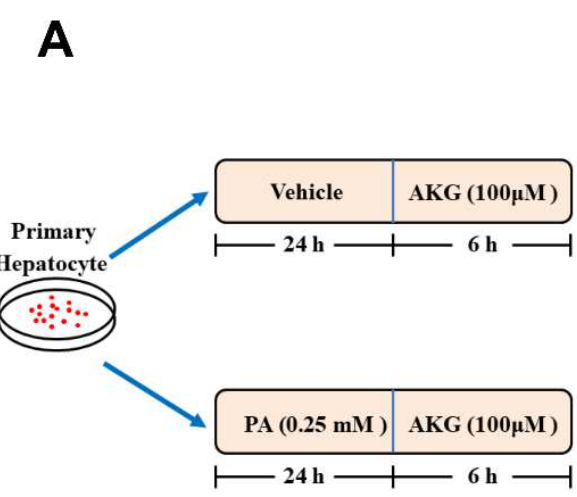

B

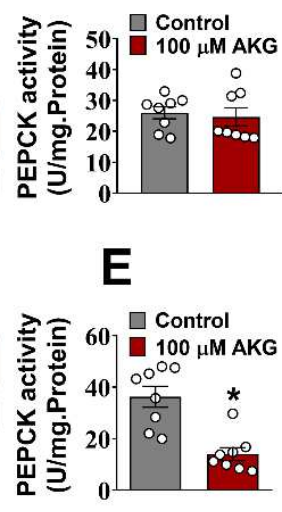

H

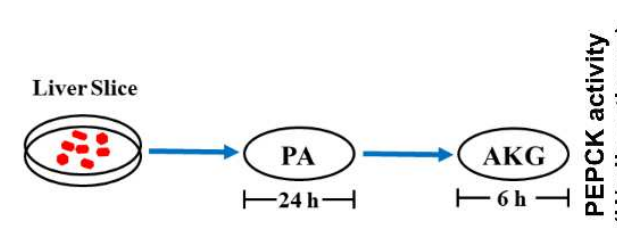

I
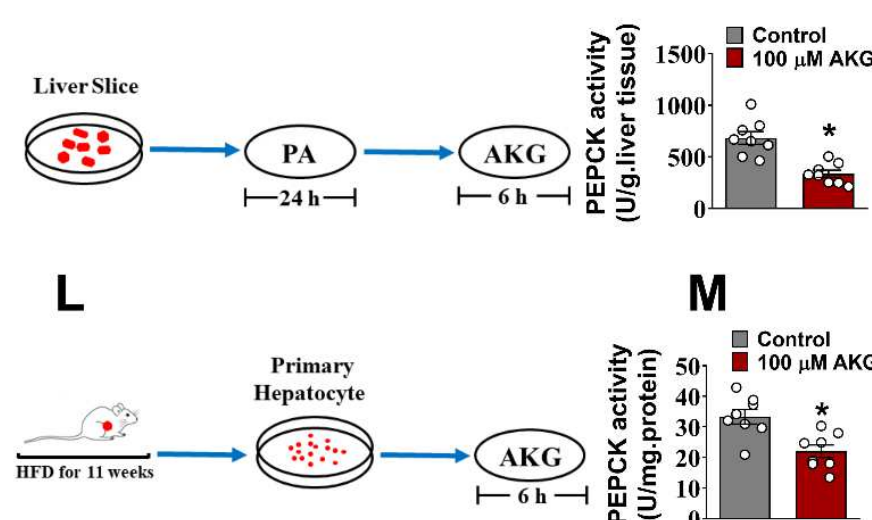

\section{M \\ M}

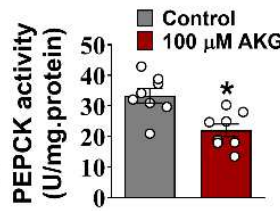

C

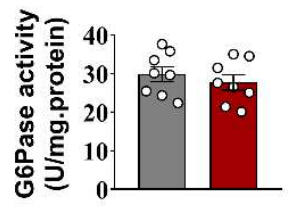

F

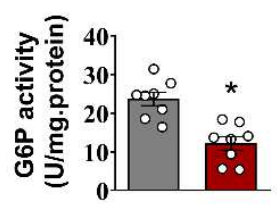

J

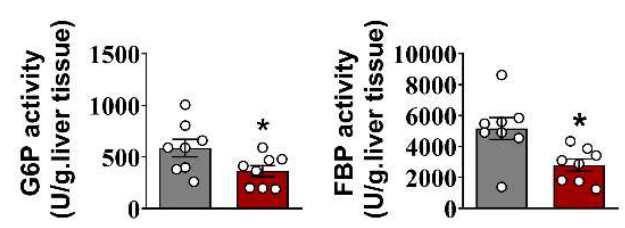

N

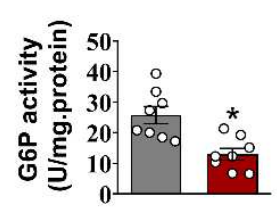

D

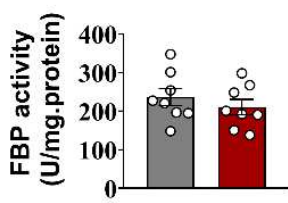

G

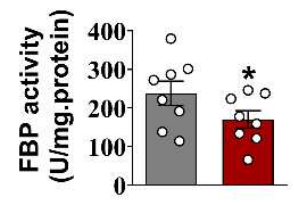

\section{K}

\section{0}

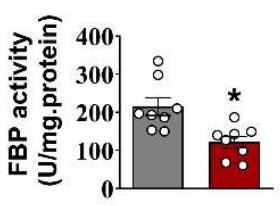

827 (A). Schematic representation of primary hepatocyte treated with AKG. 10 weeks of male C57BL/6 mice primary hepatocyte were cultured with vehicle or $0.25 \mathrm{mM}$ PA for $24 \mathrm{~h}$, then treated with vehicle or $100 \mu \mathrm{M}$ AKG for 6 hrs ( $n=8$ per group). (B-D). The activity of PEPCK (B), G6Pase (C), and FBP (D) of primary hepatocyte. Primary hepatocytes were treated with vehicle or $100 \mu \mathrm{M}$ AKG for $6 \mathrm{hrs}(\mathrm{n}=8$ per group). (E-G). The activity of PEPCK (E), G6Pase (F), and FBP (G) of primary hepatocyte. Primary hepatocyte were cultured with vehicle or $0.25 \mathrm{mM}$ PA for $24 \mathrm{hrs}$ and then treated with vehicle or $100 \mu \mathrm{M}$ AKG for $6 \mathrm{hrs}$ ( $n=8$ per group)

(H). Schematic representation of liver slice treated with AKG. C57BL/6 male mice were fed a chow diet at 10 weeks of age. Liver slices were cultured with $0.25 \mathrm{mM}$ PA for $24 \mathrm{hrs}$ and then treated with vehicle or 100 $\mu \mathrm{M}$ AKG for $6 \mathrm{hrs}(\mathrm{n}=8$ per group). (I-K). The activity of PEPCK (I), G6Pase (J), and FBP (K) of liver ( $n=8$ per group). 
839 (L). Schematic representation of primary hepatocyte treated with AKG. Ten-week-old C57BL/6 male mice 840 were fed HFD for 11 weeks. Primary hepatocytes were treated with vehicle or $100 \mu$ M AKG for 6 hrs $(n=8$ 841 per group).

842 (M-O). The activity of PEPCK (M), G6Pase (N), and FBP $(\mathrm{O})$ of primary hepatocyte ( $\mathrm{n}=8$ per group).

843 Data information: Results are presented as mean \pm SEM. In (E-G), (I-K), and (M-O), ${ }^{*} \mathrm{p} \leq 0.05$ by non-paired 844 Student's t-test.

845 


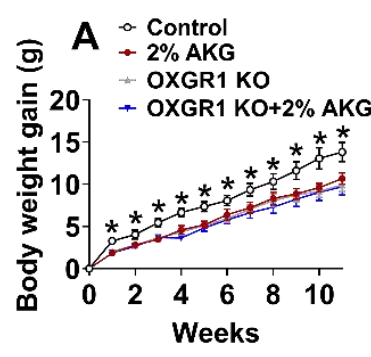

E

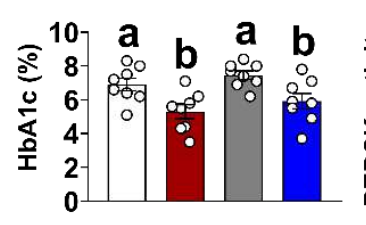

B

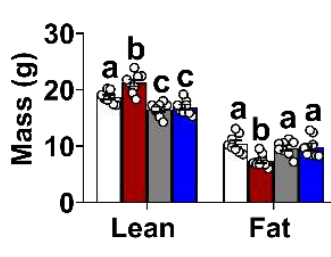

F

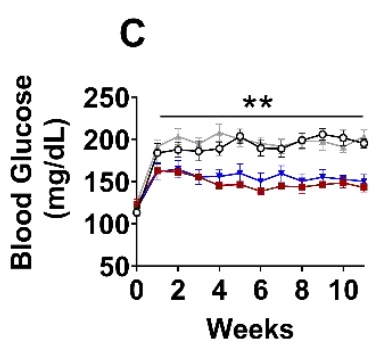

G

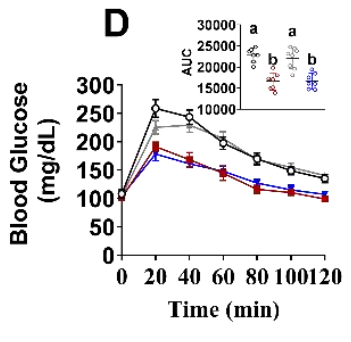

H
869

(A-C). Body weight gain (A), body composition (B), and blood glucose (C) of male WT control (littermates) or OXGR1KO mice. At 12 weeks of age, both control and KO mice were switched to HFD and further divided into two groups, receiving tap water or water supplemented with $2 \%$ AKG for 11 weeks $(\mathrm{n}=8$ per group).

(D). Pyruvate tolerance test (PTT, $1 \mathrm{~g} / \mathrm{kg}$ ). At 12 weeks of age, both control and $\mathrm{KO}$ mice were switched to HFD and further divided into two groups, receiving tap water or water supplemented with 2\% AKG for 11 weeks ( $\mathrm{n}=8$ per group).

(E). Serum HbA1c level. At 12 weeks of age, both control and KO mice were switched to HFD and further divided into two groups, receiving tap water or water supplemented with $2 \%$ AKG for 11 weeks $(n=8$ per group).

(F-H). The activity of PEPCK (F), G6Pase (G), and FBP (H) in the liver. At 12 weeks of age, both control and $\mathrm{KO}$ mice were switched to HFD and further divided into two groups, receiving tap water or water supplemented with $2 \%$ AKG for 11 weeks ( $n=8$ per group).

Data information: Results are presented as mean \pm SEM. In $(A, C), * p \leq 0.05$ by two-way ANOVA followed by post-hoc Bonferroni tests. In (D-H), different letters between bars indicate $\mathrm{p} \leq 0.05$ by one-way ANOVA followed by post-hoc Tukey’s tests. 
A

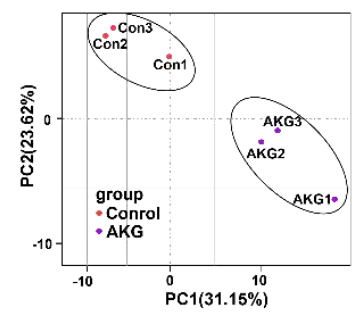

E
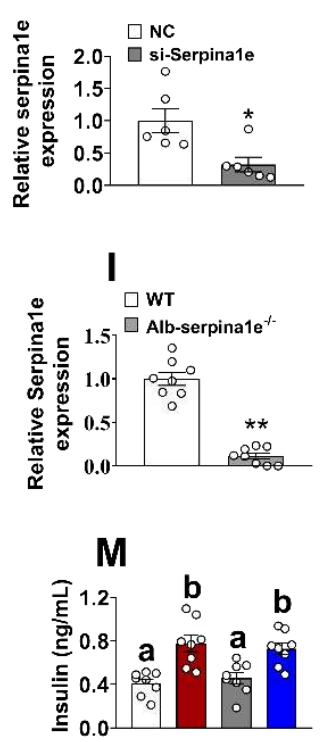
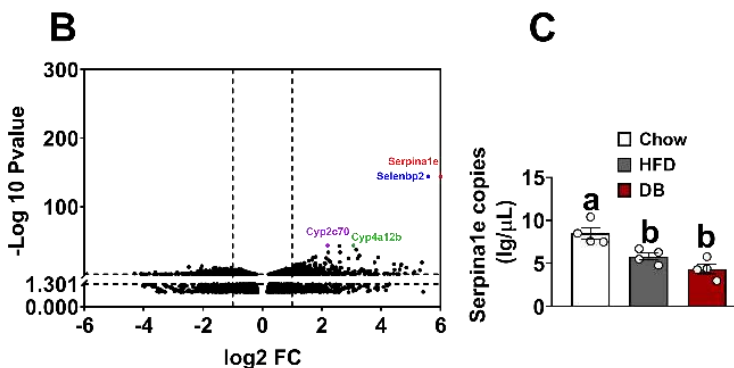

G
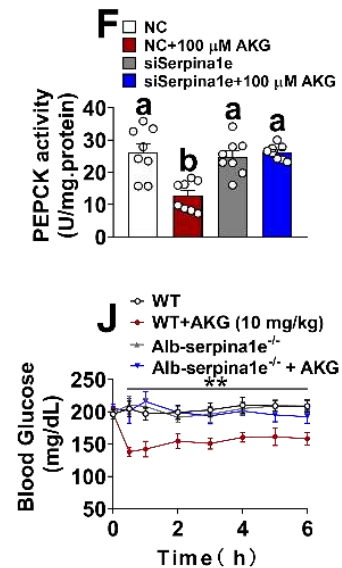

$\mathbf{N}$

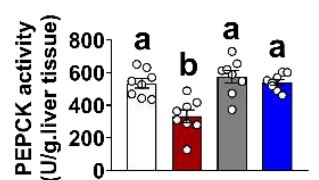

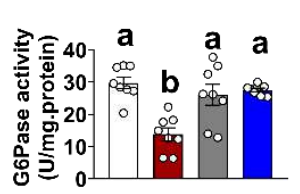

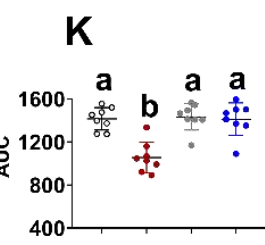

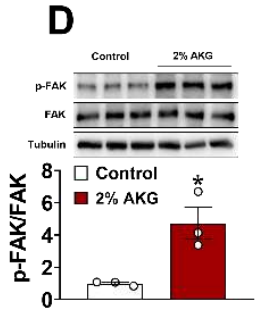

H

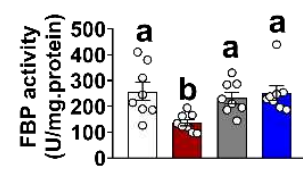

\section{L}

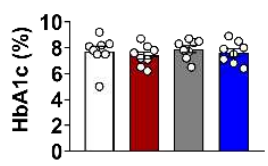

890

891 (A). Principal coordinate analysis plot ( $\mathrm{n}=3$ per group).

(B). Volcano plot of AKG-induced transcriptome signature. Genes with $\log _{2} \mathrm{FC} \geq 1$ and $-\log _{10} \mathrm{P}$ value $\geq 1.3$ were considered significant. Serpina1e (red dots), selenbp2 (blue dots), cyp2c70 (purple dots), and cyp4a12b (green dots) genes were most significantly different between groups. At 12 weeks of age, mice were switched to HFD and received tap water or water supplemented with $2 \%$ AKG for 11 weeks ( $n=3$ per group). (C). mRNA expression of serpinale in the liver tissue. Eight-week-old C57BL/6 male mice were divided into two groups and then fed a chow diet or HFD for 12 weeks. Eight-week-old db/db mice (DB) were fed a chow diet for 12 weeks ( $n=4$ per group).

(D). Immunoblots and quantification of p-FAK protein expression in liver. At 12 weeks of age, mice were switched to HFD and received tap water or water supplemented with $2 \%$ AKG for 11 weeks ( $n=3$ per group). (E). mRNA expression of serpinale in primary hepatocyte. Primary hepatocyte were transfected with negative control (NC) siRNA or si-serpinale for $24 \mathrm{hrs}(\mathrm{n}=6$ per group). (F-H). The activity of PEPCK (F), G6Pase (G), and FBP (H) in primary hepatocyte (PA treatment) cultured 
with vehicle $+\mathrm{NC}$, vehicle + si-serpinale, AKG $(100 \mu \mathrm{M})+\mathrm{NC}$, or AKG + si-serpinale for $6 \mathrm{~h}(\mathrm{n}=8$ per group).

(I). mRNA expression of serpina1e in liver ( $\mathrm{n}=8$ per group). Alb-Cre mice were crossed with LSL-Cas9EGFP mice to generate Alb-Cre/LSL-Cas9-EGFP (Alb-Cas9), a mouse model with Cas9 selectively overexpressed in Alb positive liver cells. Six-week-old male Alb-Cas9 mice were i.p injected with AAV-sgRNAs-serpina1e $\left(1 \times 10^{12} \mathrm{GC} / \mathrm{ml}\right)$ to generate a liver-specific sepinale deletion mouse model (Alb-serpina1e $\mathrm{e}^{--}$).

(J-K). Blood glucose concentration-time profile (J-K) obtained from male WT control (littermates) or Albserpina1 $^{-/-}$mice (10 weeks) fed with HFD i.p saline or AKG $(10 \mathrm{mg} / \mathrm{kg}$ body weight). The blood glucose was tested at $0,0.5,1,2,3,4,5$, and 6 hrs after injection ( $\mathrm{n}=8$ per group).

(L-M). Serum HbA1c level (L) and insulin level (M) in male WT control (littermates) or Alb-serpina1e e- $^{-/}$ mice (10 weeks) fed with HFD i.p saline or AKG (10 mg/kg body weight) at 0, 0.5, 1, 2, 3, 4, 5, and 6 hrs after injection ( $\mathrm{n}=8$ per group).

(N-P). The activity of PEPCK (N), G6Pase (O), and FBP (P) in the liver of male WT control (littermates) or Alb-serpina1 $\mathrm{e}^{-/}$mice (10 weeks) fed with HFD i.p saline or AKG (10 mg/kg body weight) at $0,0.5,1,2,3$, 4,5 , and 6 hrs after injection ( $\mathrm{n}=8$ per group).

Data information: Results are presented as mean \pm SEM. In $(\mathrm{C}),(\mathrm{F}-\mathrm{H}),(\mathrm{K})$, and $(\mathrm{M}-\mathrm{P})$, different letters between bars indicate $\mathrm{p} \leq 0.05$ by one-way ANOVA followed by post-hoc Tukey's tests. In (D-E) and (I-J), ${ }^{*} \mathrm{p} \leq 0.05$ by non-paired Student's t-test.

3

4

5

6

7


A

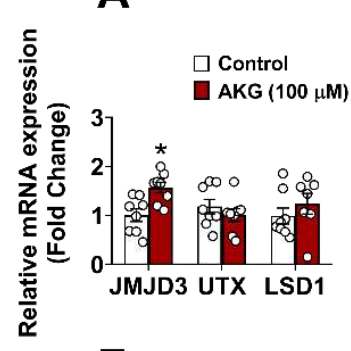

E

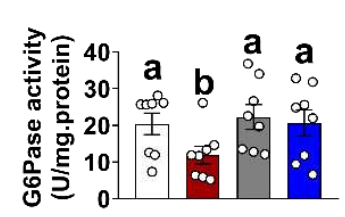

I

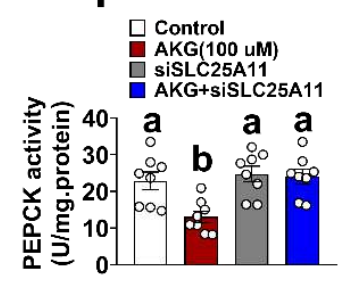

B

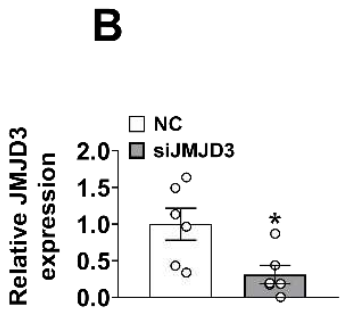

F

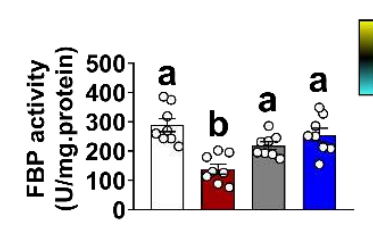

J

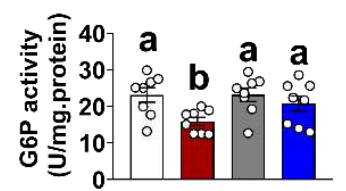

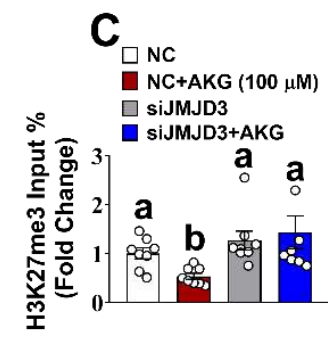

G

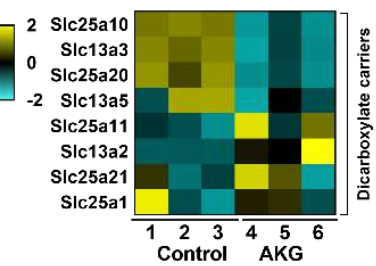

K

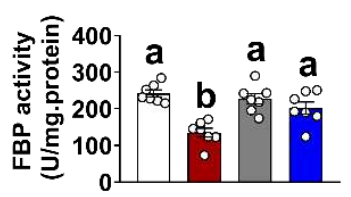

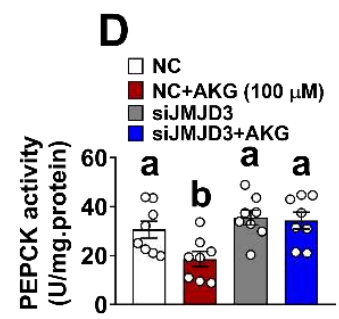

H
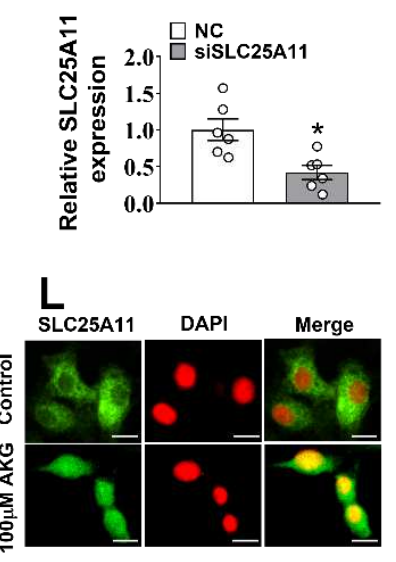

(A). mRNA expression of JMJD3, LSD, and UTX in primary hepatocyte. Primary hepatocytes were cultured with $0.25 \mathrm{mM}$ PA for $24 \mathrm{hrs}$ and then treated with vehicle or $100 \mu \mathrm{M} \mathrm{AKG} \mathrm{for} 6 \mathrm{hrs}(\mathrm{n}=8$ per group).

(B). mRNA expression of JMJD3 in primary hepatocyte. Primary hepatocytes were transfected with NC siRNA or si-SLC25A11 for $24 \mathrm{hrs}$ ( $\mathrm{n}=6$ per group).

(C). Chromatin-immunoprecipitation (ChIP) analysis of H3K27me3 in promoter of serpinale in primary hepatocyte (PA treatment) cultured with vehicle $+\mathrm{NC}$, vehicle + si-JMJD3, AKG $(100 \mu \mathrm{M})+\mathrm{NC}$, or AKG + si-JMJD3 for 6 hrs ( $\mathrm{n}=8$ per group).

(D-F). The activity of PEPCK (D), G6Pase (E), and FBP (F) in primary hepatocyte (PA treatment) cultured with vehicle $+\mathrm{NC}$, vehicle + si-JMJD3, AKG $(100 \mu \mathrm{M})+\mathrm{NC}$, or AKG + si-JMJD3 for $6 \mathrm{hrs}(\mathrm{n}=8$ per group).

(G). Relative changes of dicarboxylate carriers in response to AKG treatment. Heat maps show changes of dicarboxylate carriers in the mice liver. At 12 weeks of age, mice were switched to HFD and received tap water or water supplemented with $2 \%$ AKG for 11 weeks ( $n=3$ per group).

(H). mRNA expression of SLC25A11 in primary hepatocyte. Primary hepatocytes were treated with NC siRNA or si-serpinale ( $n=6$ per group). 
950 (I-K). The activity of PEPCK (I), G6Pase (J), and FBP (K) in primary hepatocyte (PA treatment) cultured 951 with vehicle $+\mathrm{NC}$, vehicle + si-SCL25A11, AKG $(100 \mu \mathrm{M})+\mathrm{NC}$, or AKG + si-SLC25A11 for 6 hrs $(\mathrm{n}=8$ 952 per group).

953 (L). Immunofluorescence of SLC25A11 translocation in primary hepatocyte. Primary hepatocytes were 954 cultured with $0.25 \mathrm{mM}$ PA for $24 \mathrm{~h}$ and then treated with vehicle or $100 \mu \mathrm{M}$ AKG for $6 \mathrm{hrs}(\mathrm{n}=8$ per group).

955 Scale bars, $50 \mu \mathrm{m}$.

956 Data information: Results are presented as mean \pm SEM. In (A-B) and (H) * $\mathrm{p} \leq 0.05$ by non-paired Student's 957 t-test. In (C-F) and (I-K), different letters between bars indicate $\mathrm{p} \leq 0.05$ by one-way ANOVA followed by 958 post-hoc Tukey's tests.

959

960

961

962

963

964

965

966

967

968

969

970

971

972 

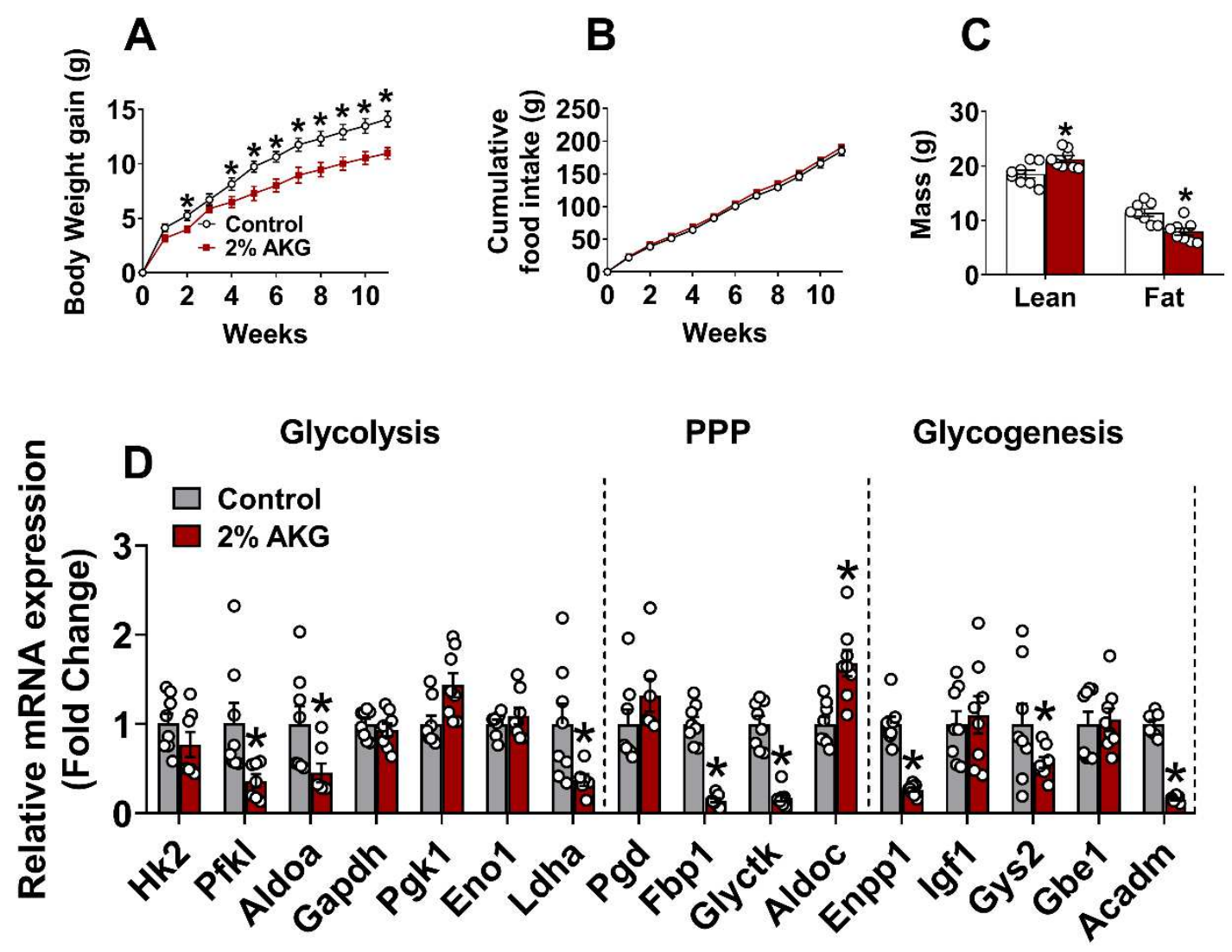

975

976 (A-C). Body weight gain (A), food intake (B), and body composition (C) of male C57BL/6 mice. At 12 weeks of age, mice were fed with HFD and received tap water or water supplemented with 2\% AKG for 11 weeks ( $\mathrm{n}=8$ per group). (D). mRNA expression of glycolysis, pentose phosphate pathway (PPP) and glycogenesis related enzymes of mice liver. At 12 weeks of age, mice were fed with HFD and received tap water or water supplemented with $2 \%$ AKG for 11 weeks ( $\mathrm{n}=8$ per group). HK2 (Hexokinase 2), phosphofructokinase (Pfkl), aldolase

(Gapdh), phosphoglycerate kinase 1 (Pgk1), enolase 1 (Eno1), lactate dehydrogenase A (Ldha), phosphogluconate dehydrogenase (Pgd), fructose-bisphosphatase 1 (Fbp1), glycerate kinase (Glyctk), fructose-bisphosphate C aldolase (Aldoc), phosphodiesterase 1 (Enpp1), insulin like growth factor 1 
986 (Igf1), glycogen synthase 2 (Gys2), 1.4-alpha-glucan branching enzyme 1 (Gbe1), acyl-CoA 987 dehydrogenase (Acadm).

988 Data information: Results are presented as mean \pm SEM. In (A), $* \mathrm{p} \leq 0.05$ by two-way ANOVA followed by 989 post-hoc Bonferroni tests. In (C and $\mathrm{E}),{ }^{*} \mathrm{p} \leq 0.05$ by non-paired Student's t-test.

990

991

992

993

994

995

996

997

998

999

1000

1001

1002

1003

1004

1005

1006

1007

1008

1009

1010

1011

1012

1013

1014 
1016 Figure S2. Acute AKG administration prevents hyperglycemia in T1D and db/db diabetic mice.

A

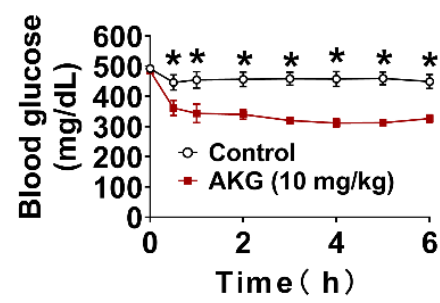

D

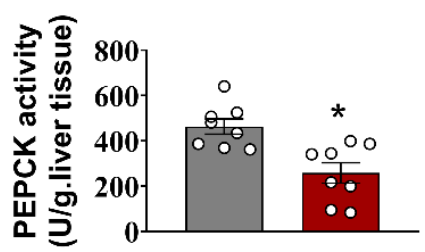

G

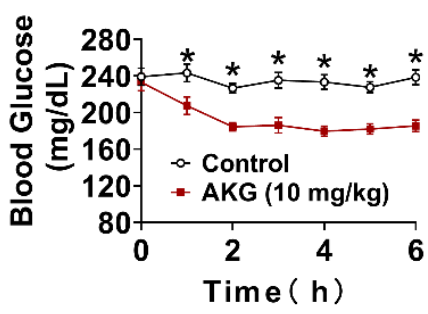

$\mathbf{J}$

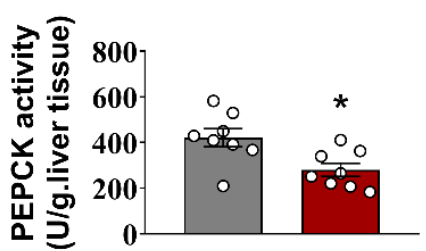

B

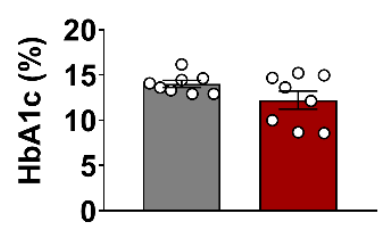

E

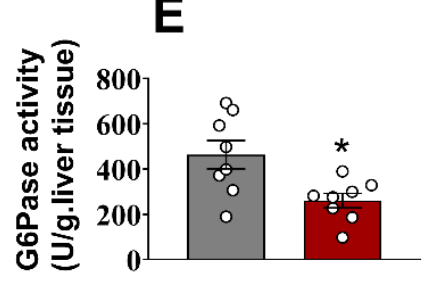

H

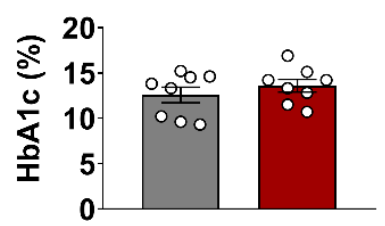

$\mathrm{K}$

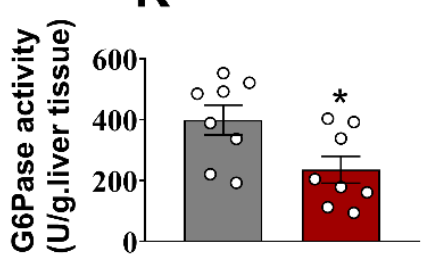

C
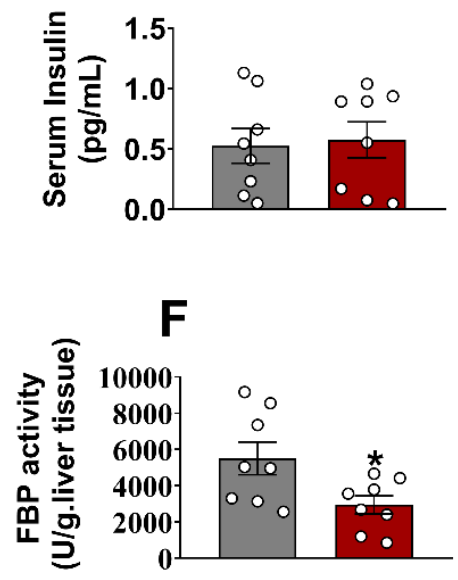

I

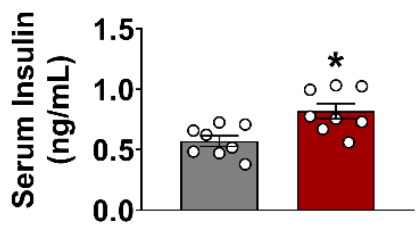

1017

1018 (A). Blood glucose concentration-time profile obtained from male mice with T1D (10 weeks) fed with chow1019 diet i.p saline or AKG (10 mg/kg body weight). The blood glucose was tested at $0,0.5,1,2,3,4,5$, and 6 $1020 \mathrm{hrs}(\mathrm{n}=8$ per group $)$.

1021 (B-C). Serum HbA1c level (B) and insulin level (C) in male mice with T1D (10 weeks) fed with chow-diet 1022 i.p saline or AKG (10 mg/kg body weight). The blood glucose was tested at $0,0.5,1,2,3,4,5$, and $6 \mathrm{hrs}$ (n $1023=8$ per group).

1024 (D-F). The activity of PEPCK (D), G6Pase (E), and FBP (F) in the liver of male mice with T1D (10 weeks) 1025 fed with chow-diet i.p saline or AKG (10 mg/kg body weight). The blood glucose was tested at 0, 0.5, 1, 2, 1026 $3,4,5$, and 6 hrs ( $\mathrm{n}=8$ per group). 
1027 (G). Blood glucose concentration-time profile obtained from male $\mathrm{db} / \mathrm{db}$ mice (10 weeks) fed with chow1028 diet i.p saline or AKG (10 mg/kg body weight). The blood glucose was tested at $0,0.5,1,2,3,4,5$, and 6 1029 hrs ( $\mathrm{n}=8$ per group $)$.

1030 (H-I). Serum HbA1c level (H) and insulin level (I) in male db/db mice (10 weeks) fed with chow-diet i.p 1031 saline or AKG (10 $\mathrm{mg} / \mathrm{kg}$ body weight) ( $\mathrm{n}=8$ per group)

1032 (J-L). The activity of PEPCK (J), G6Pase (K), and FBP (L) in the liver of male db/db mice (10 weeks) fed 1033 with chow-diet i.p saline or AKG (10 mg/kg body weight) ( $\mathrm{n}=8$ per group).

1034 Data information: Results are presented as mean \pm SEM. In (A) and (G) $* \mathrm{p} \leq 0.05$ by two-way ANOVA 1035 followed by post-hoc Bonferroni tests. In (D-F) and (I-L), * $\mathrm{p} \leq 0.05$ by non-paired Student's t-test.

1036

1037

1038

1039

1040

1041

1042

1043

1044

1045

1046

1047

1048

1049

1050

1051

1052

1053

1054

1055 
1057 Figure S3. Selenbp2, cyp2c70a, and cyp4a12b are not required for the inhibitory effects of AKG on hepatic gluconeogenesis.
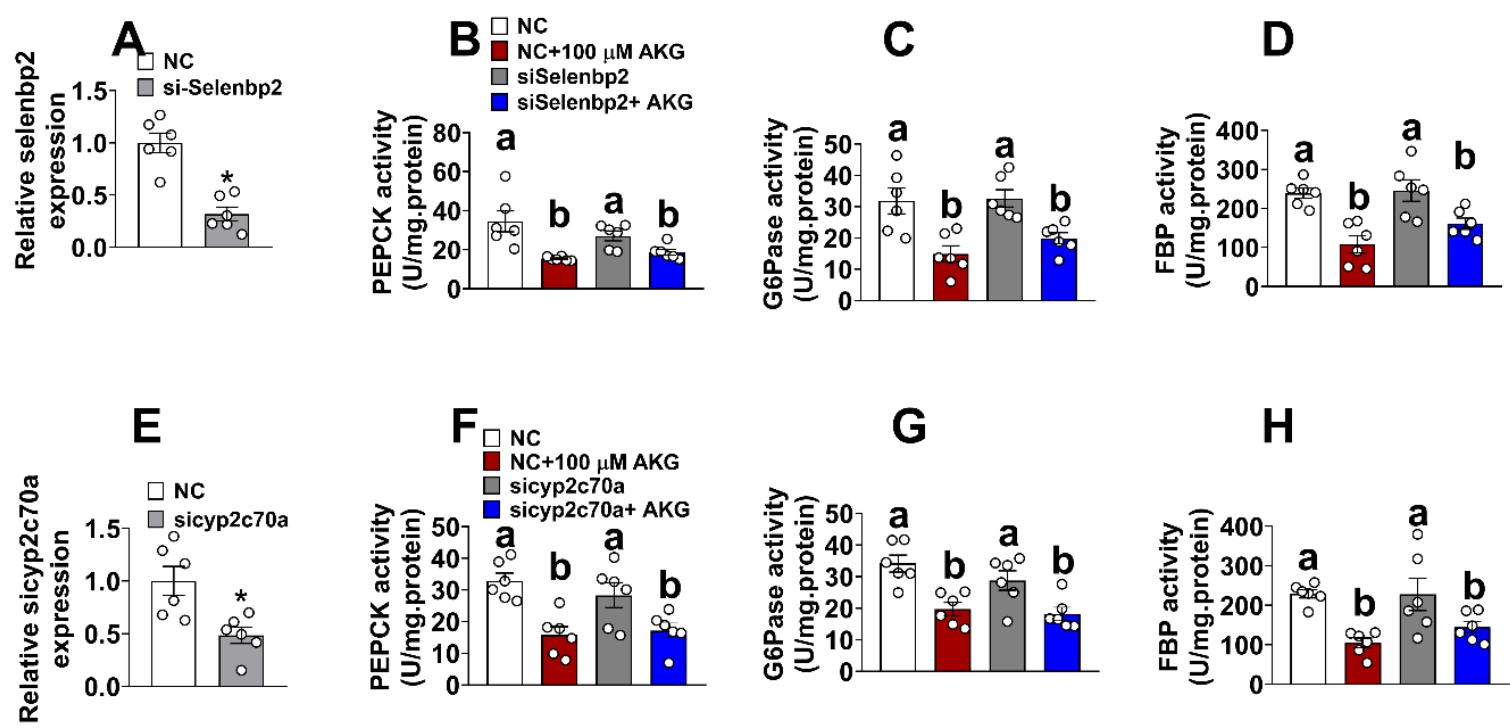

\section{H}
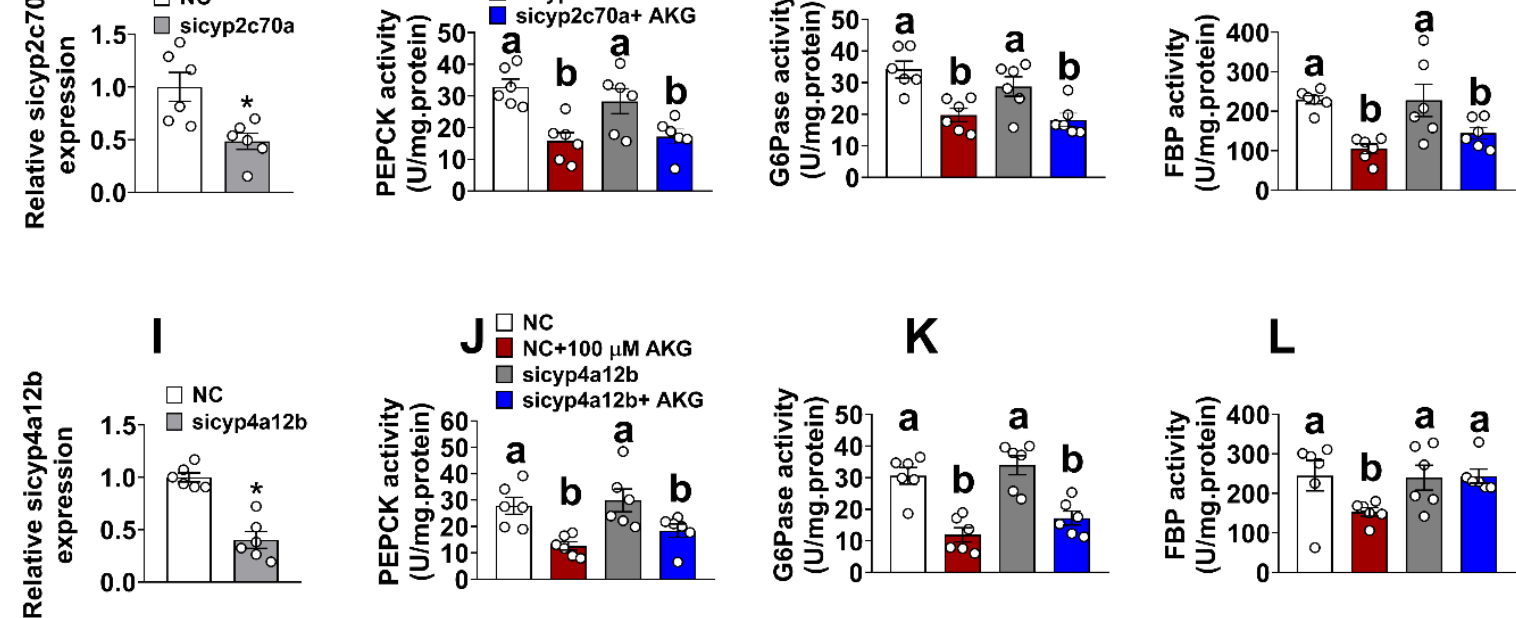

1059

1060

1061

(A). mRNA expression of selenbp2 in primary hepatocyte. Primary hepatocytes were treated with NC siRNA or si-selenbp2 ( $\mathrm{n}=6$ per group).

1062 (B-D). The activity of PEPCK (B), G6Pase (C), and FBP (D) in primary hepatocyte cells (PA treatment) cultured with vehicle $+\mathrm{NC}$, vehicle + si-selenbp2, AKG $(100 \mu \mathrm{M})+\mathrm{NC}$, or AKG + si-selenbp2 for 6 hrs $(\mathrm{n}$ $=6$ per group).

1065

(E). mRNA expression of cyp2c70a in primary hepatocyte. Primary hepatocytes were transfected with NC siRNA or si-cyp2c70a for $24 \mathrm{hrs}(\mathrm{n}=6$ per group). $(\mathrm{F}-\mathrm{H})$. The activity of PEPCK $(\mathrm{F}), \mathrm{G} 6 \mathrm{Pase}(\mathrm{G})$, and FBP $(\mathrm{H})$ in primary hepatocyte (PA treatment) cultured with vehicle $+\mathrm{NC}$, vehicle + si-cyp2c70a, AKG $(100 \mu \mathrm{M})+\mathrm{NC}$, or AKG + si-cyp2c70a for $6 \mathrm{hrs}(\mathrm{n}=6$ per group).

1070

(I). mRNA expression of cyp4a12b in primary hepatocyte. Primary hepatocytes were treated with NC siRNA or si-cyp4a12b ( $\mathrm{n}=6$ per group). 
1072 (J-L). The activity of PEPCK (J), G6Pase (K), and FBP (L) in primary hepatocyte (PA treatment) cultured 1073 with vehicle $+\mathrm{NC}$, vehicle + si-cyp4a12b, AKG $(100 \mu \mathrm{M})+\mathrm{NC}$, or AKG + si-cyp4a12b for 6 hrs $(\mathrm{n}=6$ per 1074 group).

1075 Data information: Results are presented as mean \pm SEM. In (A), (E), and (I) * $p \leq 0.05$ by non-paired Student's 1076 t-test. In (B-D), (F-H), and (J-L), different letters between bars indicate $\mathrm{p} \leq 0.05$ by one-way ANOVA 1077 followed by post-hoc Tukey's tests.

1078

1079

1080

1081

1082

1083

1084

1085

1086

1087

1088

1089

1090

1091

1092

1093

1094

1095

1096

1097

1098

1099

1100 
1102 Figure S4. Effects of AKG on RNA methylase and SLC13A2 pathway.

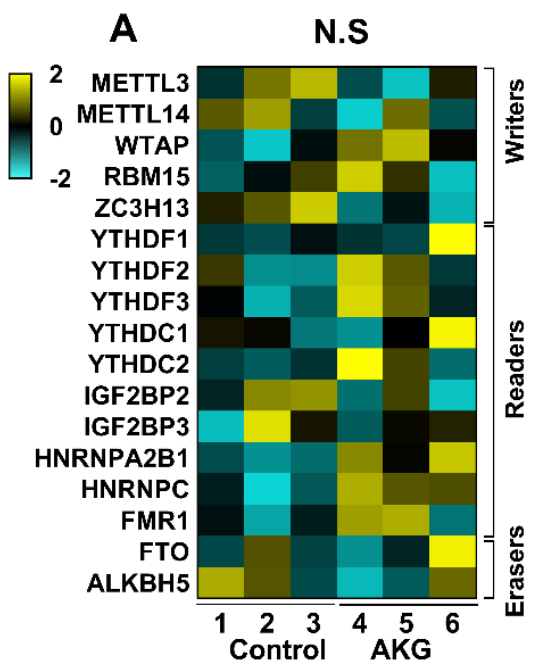

B
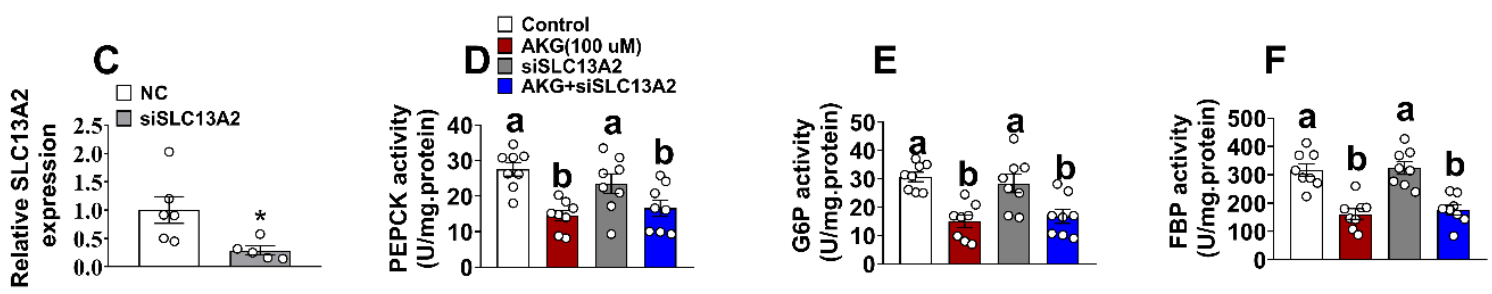

1103

1104

1105

1106

1107

1108

1109

1110

1111

1112

1113

1114

1115

1116

1117

1118

(A). Relative writers, readers, and erasers of m6A methylase gene expression in the liver by transcriptomics. At 12 weeks of age, mice were fed HFD and received tap water or water supplemented with $2 \%$ AKG for 11 weeks ( $n=3$ per group). Specifically, these writers include methyltransferase like 3 (METTL3), methyltransferase like 14 (METTL14), wilms' tumour 1- associating protein (WTAP), RNA binding motif protein 15 (RBM15), and zinc finger CCCH-type containing 13 (ZC3H13). Readers include YTH N6-methyladenosine RNA binding protein 1(YTHDF1), YTH N6-methyladenosine RNA binding protein 2 (YTHDF2), YTH N6-methyladenosine RNA binding protein 3 (YTHDF3), YTH domain containing 1 (YTHDC1), YTH domain containing 2 (YTHDC2), insulin like growth factor 2 mRNA binding protein 2 (IGF2BP2), insulin like growth factor 2 mRNA binding protein 3 (IGF2BP3), heterogeneous nuclear ribonucleoprotein A2/B1 (HNRNPA2B1), heterogeneous nuclear ribonucleoprotein C (HNRNPC), and FMRP translational regulator 1 (FMR1). Erasers include FTO alpha-ketoglutarate dependent dioxygenase (FTO) and ALKB homolog 5, RNA demethylase (ALKBH5).

(B). Relative mRNA gene expression in primary hepatocyte. Primary hepatocytes were cultured with 0.25 $\mathrm{mM}$ PA for $24 \mathrm{hrs}$ and then treated with vehicle or $100 \mu \mathrm{M}$ AKG for $6 \mathrm{hrs}(\mathrm{n}=8$ per group). 
1119 (C). mRNA expression of SLC13A2 in primary hepatocyte. Primary hepatocytes were treated with NC 1120 siRNA or si-SLC13A2 ( $\mathrm{n}=6$ per group).

1121 (D-F). The activity of PEPCK (D), G6Pase (E), and FBP (F) in primary hepatocyte cells (PA treatment) 1122 cultured with vehicle $+\mathrm{NC}$, vehicle + si-SCL13A2, AKG $(100 \mu \mathrm{M})+\mathrm{NC}$, or AKG + si-SLC13A2 for 6 hrs $1123 \quad(\mathrm{n}=8$ per group $)$.

1124 Data information: Results are presented as mean \pm SEM. In $(\mathrm{C}),{ }^{*} \mathrm{p} \leq 0.05$ by non-paired Student's t-test. In 1125 (D-F), different letters between bars indicate $\mathrm{p} \leq 0.05$ by one-way ANOVA followed by post-hoc Tukey's 1126 tests.

1127

1128

1129

1130

1131

1132

1133

1134

1135

1136

1137

1138

1139

1140

1141

1142

1143

1144

1145

1146

1147 


\section{References}

$1150 \quad$ 1. Zimmet, P., Shi, Z., El-Osta, A. \& Ji, L. Epidemic T2DM, early development and epigenetics: implications

2. Kirwan, J. P., Sacks, J. \& Nieuwoudt, S. The essential role of exercise in the management of type 2

3. Rai, M. \& Demontis, F. Systemic Nutrient and Stress Signaling via Myokines and Myometabolites. Annu

4. Boesche, K. E. \& Donkin, S. S. Pretreatment with saturated and unsaturated fatty acids regulates fatty

5. Mills, E. L. et al. Accumulation of succinate controls activation of adipose tissue thermogenesis. Nature 560, 102-106, doi:10.1038/s41586-018-0353-2 (2018). of the Chinese Famine. Nature reviews. Endocrinology 14, 738-746, doi:10.1038/s41574-018-0106-1 (2018). acid oxidation in Madin-Darby bovine kidney cells. Journal of dairy science, doi:10.3168/jds.2020-18802 (2020).

Dankel, S. J. et al. Do metabolites that are produced during resistance exercise enhance muscle hypertrophy? European journal of applied physiology 117, 2125-2135, doi:10.1007/s00421-017-3690-1 (2017).

7. Yuan, Y. et al. Exercise-induced alpha-ketoglutaric acid stimulates muscle hypertrophy and fat loss through OXGR1-dependent adrenal activation. EMBO J, e103304, doi:10.15252/embj.2019103304 (2020).

8. He, L. et al. Alpha-ketoglutarate suppresses the NF-KB-mediated inflammatory pathway and enhances the PXR-regulated detoxification pathway. Oncotarget 8, 102974-102988, doi:10.18632/oncotarget.16875 (2017).

9. $\mathrm{He}$, L. et al. Prevention of Oxidative Stress by $\alpha$-Ketoglutarate via Activation of CAR Signaling and Modulation of the Expression of Key Antioxidant-Associated Targets in Vivo and in Vitro. Journal of agricultural and food chemistry 66, 11273-11283, doi:10.1021/acs.jafc.8b04470 (2018).

10. Mangal, J. L. et al. Metabolite releasing polymers control dendritic cell function by modulating their energy metabolism. Journal of materials chemistry. B 8, 5195-5203, doi:10.1039/d0tb00790k (2020).

11. Bhagat, T. D. et al. Lactate-mediated epigenetic reprogramming regulates formation of human pancreatic cancer-associated fibroblasts. eLife 8, doi:10.7554/eLife.50663 (2019).

12. Morris, J. P. t. et al. alpha-Ketoglutarate links p53 to cell fate during tumour suppression. Nature 573, 595-599, doi:10.1038/s41586-019-1577-5 (2019).

13. Cai, X. et al. $\alpha$-Ketoglutarate prevents skeletal muscle protein degradation and muscle atrophy through PHD3/ADRB2 pathway. FASEB journal : official publication of the Federation of American Societies for Experimental Biology 32, 488-499, doi:10.1096/fj.201700670R (2018).

14. Yuan, Y. et al. Exercise-induced alpha-ketoglutaric acid stimulates muscle hypertrophy and fat loss through OXGR1-dependent adrenal activation. The EMBO journal 39, e103304, doi:10.15252/embj.2019103304 (2020).

15. Tian, Q. et al. Dietary alpha-ketoglutarate promotes beige adipogenesis and prevents obesity in middleaged mice. Aging cell 19, e13059, doi:10.1111/acel.13059 (2020).

16. Tekwe, C. D. et al. Oral administration of $\alpha$-ketoglutarate enhances nitric oxide synthesis by endothelial cells and whole-body insulin sensitivity in diet-induced obese rats. Experimental biology and medicine (Maywood, N.J.) 244, 1081-1088, doi:10.1177/1535370219865229 (2019). 
17. Dick, B. P., Yousif, A., Raheem, O. \& Hellstrom, W. J. G. Does Lowering Hemoglobin A1c Reduce Penile Prosthesis Infection: A Systematic Review. Sexual medicine reviews, doi:10.1016/j.sxmr.2020.06.004 (2020).

18. Berton, R. et al. Metabolic time-course response after resistance exercise: A metabolomics approach. J Sports Sci 35, 1211-1218, doi:10.1080/02640414.2016.1218035 (2017).

19. Ding, L., Xu, Y., Liu, S., Bi, Y. \& Xu, Y. Hemoglobin A1c and diagnosis of diabetes. Journal of diabetes 10, 365-372, doi:10.1111/1753-0407.12640 (2018).

20. Han, H. S., Kang, G., Kim, J. S., Choi, B. H. \& Koo, S. H. Regulation of glucose metabolism from a livercentric perspective. Experimental \& molecular medicine 48, e218, doi:10.1038/emm.2015.122 (2016).

21. Hoang, M. \& Joseph, J. W. The role of $\alpha$-ketoglutarate and the hypoxia sensing pathway in the regulation of pancreatic $\beta$-cell function. Islets 12, 108-119, doi:10.1080/19382014.2020.1802183 (2020).

22. Chantzichristos, D., Eliasson, B. \& Johannsson, G. MANAGEMENT OF ENDOCRINE DISEASE: Disease burden and treatment challenges in patients with both Addison's disease and type 1 diabetes mellitus. European journal of endocrinology, doi:10.1530/eje-20-0052 (2020).

23. Barnett, R. Type 1 diabetes. Lancet (London, England) 391, 195, doi:10.1016/s0140-6736(18)30024-2 (2018).

24. Xiao, D. et al. The glutamine-alpha-ketoglutarate (AKG) metabolism and its nutritional implications. Amino acids 48, 2067-2080, doi:10.1007/s00726-016-2254-8 (2016).

25. Zhang, $X$. et al. Celastrol reverses palmitic acid (PA)-caused TLR4-MD2 activation-dependent insulin resistance via disrupting MD2-related cellular binding to PA. Journal of cellular physiology 233, 68146824, doi:10.1002/jcp.26547 (2018).

26. Diehl, J. et al. Expression and localization of GPR91 and GPR99 in murine organs. Cell and tissue research 364, 245-262, doi:10.1007/s00441-015-2318-1 (2016).

27. Farshchian, M. et al. Serpin peptidase inhibitor clade A member 1 (SerpinA1) is a novel biomarker for progression of cutaneous squamous cell carcinoma. The American journal of pathology 179, 1110-1119, doi:10.1016/j.ajpath.2011.05.012 (2011).

28. Tsujimoto, S. et al. Selenium-binding protein 1: its physiological function, dependence on aryl hydrocarbon receptors, and role in wasting syndrome by 2,3,7,8-tetrachlorodibenzo-p-dioxin. Biochimica et biophysica acta 1830, 3616-3624, doi:10.1016/j.bbagen.2013.03.008 (2013).

29. Straniero, S. et al. Of mice and men: murine bile acids explain species differences in the regulation of bile acid and cholesterol metabolism. Journal of lipid research 61, 480-491, doi:10.1194/jlr.RA119000307 (2020).

30. Muller, D. N. et al. Mouse Cyp4a isoforms: enzymatic properties, gender- and strain-specific expression, and role in renal 20-hydroxyeicosatetraenoic acid formation. The Biochemical journal 403, 109-118, doi:10.1042/bj20061328 (2007).

31. Taylor, E. B. Functional Properties of the Mitochondrial Carrier System. Trends in cell biology 27, 633644, doi:10.1016/j.tcb.2017.04.004 (2017).

32. Lazo-Fernandez, Y., Welling, P. A. \& Wall, S. M. $\alpha$-Ketoglutarate stimulates pendrin-dependent $\mathrm{Cl}(-)$ absorption in the mouse CCD through protein kinase C. American journal of physiology. Renal physiology 315, F7-f15, doi:10.1152/ajprenal.00576.2017 (2018).

33. Liu, P. S. et al. $\alpha$-ketoglutarate orchestrates macrophage activation through metabolic and epigenetic reprogramming. Nature immunology 18, 985-994, doi:10.1038/ni.3796 (2017).

34. Cieslar-Pobuda, A. et al. DNMT3B deficiency alters mitochondrial biogenesis and $\alpha$-ketoglutarate levels in human embryonic stem cells. Stem cells (Dayton, Ohio), doi:10.1002/stem.3256 (2020). 
35. Liang, Z., Kidwell, R. L., Deng, H. \& Xie, Q. Epigenetic N6-methyladenosine modification of RNA and DNA regulates cancer. Cancer biology \& medicine 17, 9-19, doi:10.20892/j.issn.2095-3941.2019.0347 (2020).

36. Liu, S. et al. The emerging molecular mechanism of m(6)A modulators in tumorigenesis and cancer progression. Biomedicine \& pharmacotherapy = Biomedecine \& pharmacotherapie 127, 110098, doi:10.1016/j.biopha.2020.110098 (2020).

37. Dong, Z. \& Cui, H. The Emerging Roles of RNA Modifications in Glioblastoma. Cancers 12, doi:10.3390/cancers12030736 (2020).

38. Frye, M., Harada, B. T., Behm, M. \& He, C. RNA modifications modulate gene expression during development. Science (New York, N.Y.) 361, 1346-1349, doi:10.1126/science.aau1646 (2018).

39. Seok, S. et al. Fasting-induced JMJD3 histone demethylase epigenetically activates mitochondrial fatty acid $\beta$-oxidation. The Journal of clinical investigation 128, 3144-3159, doi:10.1172/jci97736 (2018).

40. Martinez-Moreno, J. M. et al. Epigenetic Modifiers as Potential Therapeutic Targets in Diabetic Kidney Disease. International journal of molecular sciences 21, doi:10.3390/ijms21114113 (2020).

41. Yin, X., Yang, S., Zhang, M. \& Yue, Y. The role and prospect of JMJD3 in stem cells and cancer. Biomedicine $\&$ pharmacotherapy $=$ Biomedecine \& pharmacotherapie 118, 109384, doi:10.1016/j.biopha.2019.109384 (2019).

42. Osis, G. et al. Regulation of renal NaDC1 expression and citrate excretion by NBCe1-A. American journal of physiology. Renal physiology 317, F489-F501, doi:10.1152/ajprenal.00015.2019 (2019).

43. Barrosse-Antle, M. et al. A severe case of hyperinsulinism due to hemizygous activating mutation of glutamate dehydrogenase. Pediatric diabetes 18, 911-916, doi:10.1111/pedi.12507 (2017).

44. Thevis, M., Thomas, A. \& Schänzer, W. Insulin. Handbook of experimental pharmacology, 209-226, doi:10.1007/978-3-540-79088-4_10 (2010).

45. DeFronzo, R. A. Pathogenesis of type 2 diabetes mellitus. The Medical clinics of North America 88, 787835, ix, doi:10.1016/j.mcna.2004.04.013 (2004).

46. Apostolova, N. et al. Mechanisms of action of metformin in type 2 diabetes: Effects on mitochondria and leukocyte-endothelium interactions. Redox biology 34, 101517, doi:10.1016/j.redox.2020.101517 (2020).

47. Karásek, D. Pioglitazone. Vnitrni lekarstvi 66, 121-125 (2020).

48. Carrell, R. W. \& Lomas, D. A. Alpha1-antitrypsin deficiency--a model for conformational diseases. The New England journal of medicine 346, 45-53, doi:10.1056/NEJMra010772 (2002).

49. Huasong, G. et al. Serine protease inhibitor (SERPIN) B1 suppresses cell migration and invasion in glioma cells. Brain research 1600, 59-69, doi:10.1016/j.brainres.2014.06.017 (2015).

50. Zdzisinska, B., Zurek, A. \& Kandefer-Szerszen, M. Alpha-Ketoglutarate as a Molecule with Pleiotropic Activity: Well-Known and Novel Possibilities of Therapeutic Use. Arch Immunol Ther Exp (Warsz) 65, 2136, doi:10.1007/s00005-016-0406-x10.1007/s00005-016-0406-x [pii] (2017).

51. Liu, S. J. et al. FTO is a transcriptional repressor to auto-regulate its own gene and potentially associated with homeostasis of body weight. Journal of molecular cell biology 11, 118-132, doi:10.1093/jmcb/mjy028 (2019).

52. Ma, S. et al. The interplay between m6A RNA methylation and noncoding RNA in cancer. Journal of hematology \& oncology 12, 121, doi:10.1186/s13045-019-0805-7 (2019).

53. Zha, X. et al. Overexpression of METTL3 attenuates high-glucose induced RPE cell pyroptosis by regulating miR-25-3p/PTEN/Akt signaling cascade through DGCR8. Aging 12, 8137-8150, doi:10.18632/aging.103130 (2020).

54. Majumder, S. et al. Shifts in podocyte histone H3K27me3 regulate mouse and human glomerular 
disease. The Journal of clinical investigation 128, 483-499, doi:10.1172/jci95946 (2018).

55. Jambhekar, A., Dhall, A. \& Shi, Y. Roles and regulation of histone methylation in animal development. Nat Rev Mol Cell Biol 20, 625-641, doi:10.1038/s41580-019-0151-1 (2019).

56. Lee, H. W. et al. Expression of sodium-dependent dicarboxylate transporter $1(\mathrm{NaDC} 1 / \mathrm{SLC} 13 \mathrm{~A} 2)$ in normal and neoplastic human kidney. American journal of physiology. Renal physiology 312, F427-f435, doi:10.1152/ajprenal.00559.2016 (2017).

57. Takahashi, R. et al. Cell type-specific activation of metabolism reveals that beta-cell secretion suppresses glucagon release from alpha-cells in rat pancreatic islets. American journal of physiology. Endocrinology and metabolism 290, E308-316, doi:10.1152/ajpendo.00131.2005 (2006).

58. Monné, M., Miniero, D. V., lacobazzi, V., Bisaccia, F. \& Fiermonte, G. The mitochondrial oxoglutarate carrier: from identification to mechanism. Journal of bioenergetics and biomembranes 45, 1-13, doi:10.1007/s10863-012-9475-7 (2013).

59. Lash, L. H. Mitochondrial glutathione transport: physiological, pathological and toxicological implications. Chemico-biological interactions 163, 54-67, doi:10.1016/j.cbi.2006.03.001 (2006).

60. Gutiérrez-Aguilar, M. \& Baines, C. P. Physiological and pathological roles of mitochondrial SLC25 carriers. The Biochemical journal 454, 371-386, doi:10.1042/bj20121753 (2013).

61. Cappello, A. R. et al. Functional and structural role of amino acid residues in the odd-numbered transmembrane alpha-helices of the bovine mitochondrial oxoglutarate carrier. Journal of molecular biology 369, 400-412, doi:10.1016/j.jmb.2007.03.048 (2007).

62. Glick, D. et al. BNip3 regulates mitochondrial function and lipid metabolism in the liver. Molecular and cellular biology 32, 2570-2584, doi:10.1128/mcb.00167-12 (2012).

63. Zhande, R. et al. Dephosphorylation by default, a potential mechanism for regulation of insulin receptor substrate-1/2, Akt, and ERK1/2. The Journal of biological chemistry 281, 39071-39080, doi:10.1074/jbc.M605251200 (2006).

64. Zhu, C. et al. Heparin Increases Food Intake through AgRP Neurons. Cell reports 20, 2455-2467, doi:10.1016/j.celrep.2017.08.049 (2017).

65. Bookout, A. L. \& Mangelsdorf, D. J. Quantitative real-time PCR protocol for analysis of nuclear receptor signaling pathways. Nucl Recept Signal 1, e012, doi:10.1621/nrs.01012 (2003).

66. Chini, V., Foka, A., Dimitracopoulos, G. \& Spiliopoulou, I. Absolute and relative real-time PCR in the quantification of tst gene expression among methicillin-resistant Staphylococcus aureus: evaluation by two mathematical models. Lett Appl Microbiol 45, 479-484, doi:10.1111/j.1472-765X.2007.02208.x (2007).

67. Zhang, Q. et al. Inhibited expression of hematopoietic progenitor kinase 1 associated with loss of jumonji domain containing 3 promoter binding contributes to autoimmunity in systemic lupus erythematosus. Journal of autoimmunity 37, 180-189, doi:10.1016/j.jaut.2011.09.006 (2011).

68. Lee, Y. Y. et al. Loss of tumor suppressor IGFBP4 drives epigenetic reprogramming in hepatic carcinogenesis. Nucleic acids research 46, 8832-8847, doi:10.1093/nar/gky589 (2018).

69. Parkhomchuk, D. et al. Transcriptome analysis by strand-specific sequencing of complementary DNA. Nucleic acids research 37, e123, doi:10.1093/nar/gkp596 (2009). 
Table 1. Clinical characteristics of all human subjects.

\begin{tabular}{|c|c|c|c|c|c|c|c|c|}
\hline NO. & Gender & Age & $\begin{array}{l}\text { Height } \\
\text { (cm) }\end{array}$ & $\begin{array}{l}\text { Weight } \\
\text { (kg) }\end{array}$ & $\begin{array}{c}\text { HbA1c } \\
(\%)\end{array}$ & $\begin{array}{c}\mathrm{TCHO} \\
(\mathrm{mmol} / \mathrm{L})\end{array}$ & $\begin{array}{l}\text { WCF } \\
(\mathrm{cm})\end{array}$ & $\begin{array}{l}\mathrm{HCF} \\
(\mathrm{cm})\end{array}$ \\
\hline 1 & $\mathrm{M}$ & 51 & 172 & 77.5 & 8.8 & 5.52 & 86 & 98 \\
\hline 2 & M & 55 & 170 & 85.5 & 7.9 & 6.25 & 90 & 101 \\
\hline 3 & $\mathrm{M}$ & 49 & 168 & 76 & 7.1 & 7.56 & 86 & 97 \\
\hline 4 & $\mathrm{M}$ & 41 & 168.5 & 78.2 & 9.5 & 6.18 & 89 & 102 \\
\hline 5 & $\mathrm{M}$ & 58 & 183.5 & 69.8 & 6.1 & 5.05 & 83 & 94 \\
\hline 6 & M & 57 & 169.5 & 70.2 & 7.2 & 5.32 & 90 & 96 \\
\hline 7 & $\mathrm{~F}$ & 77 & / & I & 7.1 & 3.89 & I & / \\
\hline 8 & M & 71 & 162.5 & 77.3 & 8.1 & 4.29 & 96 & 102 \\
\hline 9 & M & 66 & 163 & 74.7 & 7.2 & 6.02 & 93 & 100 \\
\hline 10 & $\mathrm{M}$ & 85 & / & / & 5.9 & 6.53 & I & / \\
\hline 11 & M & 75 & 161 & 79.7 & 6.5 & 3.63 & 103 & 105 \\
\hline 12 & M & 67 & 169 & 63.3 & 5.9 & 2.66 & 82 & 90 \\
\hline 13 & M & 38 & 170.5 & 86.4 & 5.5 & 4.84 & 102 & 102 \\
\hline 14 & M & 57 & 168.5 & 78.7 & 5.9 & 4.37 & 98 & 100 \\
\hline 15 & M & 26 & 171 & 88.2 & 5.5 & 4.09 & 93 & 110 \\
\hline 16 & M & 59 & 160 & 59.3 & 6 & 5.16 & 83 & 92 \\
\hline 17 & $\mathrm{M}$ & 38 & 176.5 & 102.5 & 6.2 & 5.82 & 103 & 110 \\
\hline 18 & M & 38 & 160.5 & 61.7 & 6.5 & 5.2 & 82 & 92 \\
\hline 19 & M & 45 & 175.5 & 82.9 & 7.1 & 4.25 & 89 & 100 \\
\hline 20 & M & 54 & 169.5 & 72.8 & 6.3 & 4.03 & 88 & 94 \\
\hline 21 & M & 41 & 173.5 & 80.2 & 7.1 & 5.76 & 88 & 98 \\
\hline 22 & F & 70 & 159.5 & 53.5 & 6.6 & 5.37 & 79 & 88 \\
\hline 23 & M & 70 & 169 & 75 & 6.1 & 5.76 & 88 & 99 \\
\hline 24 & $\mathrm{~F}$ & 71 & 162 & 58.7 & 6.7 & 5.98 & 79 & 94 \\
\hline 25 & $\mathrm{M}$ & 54 & 172 & 91.1 & 7.5 & 4.6 & 109 & 108 \\
\hline 26 & M & 56 & 169.5 & 69.8 & 6.9 & 5.22 & 82 & 95 \\
\hline 27 & M & 54 & 166.5 & 64.4 & 11.7 & 5.61 & 83 & 88 \\
\hline 28 & M & 47 & 174 & 83.5 & 7.1 & 4.1 & 93 & 103 \\
\hline 29 & M & 47 & 174 & 90 & 7.5 & 5.13 & 105 & 104 \\
\hline 30 & M & 58 & 177 & 78.3 & 9.4 & 4.24 & 95 & 93 \\
\hline 31 & M & 56 & 168 & 69.5 & 7.7 & 3.88 & 86 & 90 \\
\hline 32 & $\mathrm{~F}$ & 76 & 152.5 & 63.5 & 6.6 & 4.44 & 87 & 97 \\
\hline 33 & M & 70 & 173.5 & 77.7 & 7.3 & 3.16 & 88 & 96 \\
\hline 34 & M & 55 & 168 & 73.3 & 6 & 4.76 & 88 & 99 \\
\hline 35 & M & 56 & 166.5 & 76.2 & 5.7 & 4.89 & 92 & 96 \\
\hline 36 & M & 56 & / & I & 5.6 & 3.29 & / & / \\
\hline 37 & $\mathrm{~F}$ & 50 & I & 1 & 5.9 & 4.9 & I & / \\
\hline 38 & M & 63 & 174.5 & 80 & 5.5 & 4.43 & 97 & 100 \\
\hline 39 & M & 48 & 175 & 75.2 & 5.7 & 5.79 & 80 & 98 \\
\hline 40 & M & 50 & 166.5 & 75.9 & 5.2 & 4.18 & 84 & 95 \\
\hline 41 & M & 56 & 177 & 77 & 5.7 & 5.58 & 90 & 97 \\
\hline 42 & F & 72 & 163 & 69 & 6.1 & 4.61 & 83 & 93 \\
\hline
\end{tabular}


Table 2. PCR primer sequences of related genes

\begin{tabular}{|c|c|c|}
\hline $\begin{array}{c}\text { Gene } \\
\text { abbreviation }\end{array}$ & Forward Primer $\quad\left(5^{\prime}-3^{\prime}\right)$ & Reverse Primer $\left(5^{\prime}-3^{\prime}\right)$ \\
\hline G6Pase & TCTTAAAGAGACTGTGGGCATCAA & AATACGGGCGTTGTCCAAAC \\
\hline PEPCK & TGTGTGGGCGATGACATTG & TGAGGTGCCAGGAGCAACTC \\
\hline FBP & CGGGAGATCAAGTGAAGAAGCT & CAGGTTCGACTATGATGGCATGT \\
\hline JMJD3 & CACCCCAGCAAACCATATTATGC & CACACAGCCATGCA GGG ATT \\
\hline LSD1 & TTTGGGAGTGTTGAAGCAGC & CAGAACACACGGTCAAAGCA \\
\hline HK2 & CCTGCTACAGGTCCGAGCCATCTT & GAGGATGAAGCTTGTACAGTGTCC \\
\hline PFKL & AATGTGCTGGGCCACTTGCAGCAG & TGACCGGACTGAAGGCCACTACCT \\
\hline Aldoa & ATGAGGAGATTGCCATGGCAACGG & TTTAGAGCAGAGGCCTGCAGGGCT \\
\hline GAPDH & GGGACAAGGATAGTCATTTTGGGG & TGTCATTGAGAGCAATGCCAGCCC \\
\hline PGK1 & AGACTGGCCAAGCTACTGTGGCCT & GAAGTGGCTTTCACCACCTCATCC \\
\hline ENO1 & ACCAACCCTAAGCGGATTGCCAAG & AGTCTTGATCTGCCCAGTGCAGAG \\
\hline Ldha & GCAGACAAGGAGCAGTGGAAGGAG & ACACTGAGGAAGACATCCTCATTG \\
\hline Pgd & GGACACGACAAGAAGATGCC & TCTTTGTTCCCTCCTGGCAT \\
\hline fbp1 & AGGAAGCACAAAGCCAAGTGAAGG & TGAGGATGAAGTGACCTTGGGCAT \\
\hline glyctk & GAACCCCTGTCTCACCAAGA & TGACCACATTCCCAGACCTC \\
\hline Aldoc & CTCTACATCGCCAACCATGC & CGGGTACAGCAATGTAAGGC \\
\hline Enpp1 & ACACACACGCACACATACAC & AAAGAGGACTGGGATGTGCA \\
\hline IGF1 & GTCACACAAACTCACCACCC & TTCTGATGTTGCACCCTCCT \\
\hline gys2 & GCTTGGGCGTTATCTCTGTG & GCGGTGGTATATCTGCCTCT \\
\hline Gbe1 & AGGATGTATCAGGGATGCCG & CAAGGTAGCGTCGATTGGTG \\
\hline Acadm & AAGGTTGAACTCGCTAGGCT & TCATCAGCTTCTCCACAGGG \\
\hline Mettl3 & ACTTACGCTGACCACTCCAA & TTCTGATGCTGAAGAGGCCA \\
\hline Mettl14 & TTCTGGGGAAGGATTGGACC & ACGGTTCCTTTGATCCCCAT \\
\hline WTAP & TCAGTGCGGGGTATGAAAGT & ACCTTTCCCACTCACTGCTT \\
\hline RBM15 & СТTССТСТTССТCAGCCACA & GAAGAACCCCGGTGTTTTCC \\
\hline ZC3H13 & GAAGAAGCCCGCAGTTATGG & СCTACTTCTGCCCATCCGAT \\
\hline
\end{tabular}




\begin{tabular}{lll} 
YTHDF1 & CTGCAGTTAAGACGGTGGGT & TAGCAATGGCTGCCCATGAA \\
YTHDF2 & AGCCAATGAGGAAAGGGCATT & CTCCCCAAACACAGAGACTCAA \\
YTHDF3 & TGTTCTATCTTGATTTGACTTTGCT & ATAGCTGTTATTCTGATTTGTCTGG \\
YTHDC1 & AAGCAGATCCAGCCAGTCTT & ATCTTCCTCCCCTCCTTCCT \\
YTHDC2 & GATGGATGCCTGCCTTTCTG & AACTGCTCTACTTGGCTCGT \\
IGF2BP2 & GAAAGGAGAACTCTGGGGCT & GGTTTCTGCCTTCTTTGCCA \\
IGF2BP3 & TCTGTTTATTCCCGCCCTGT & TCCCTGAGCCTTGAACTGAG \\
HNRNPA2B1 & CAGACTGTGTGGTTATGCGG & TCTTCACAGTCACATGGGCT \\
HNRNPC & TGCAGAGCCAAAAGTGAACC & TGAGTAGAGGGGACGGAGAA \\
FMR1 & GAAGAGGAAGAGGAGGAGGC & CTACGCTGTCTGGCTTTTCC \\
FTO & ATCCAGTGACAGAGACCAGC & GGGTCTTACTCCTGGCACTT \\
ALKBH5 & ACCATCAAGAAGCCCCTCTC & GCACACATATCAGGGCGAAG \\
SLC25A11 & TACTGTGTTGTTTGAGCGCC & GTTCCCACAAATGCACCAGT \\
SLC13A2 & CAGCTAGGCCTCCAGATCTC & GGAAACACACCCGGACTCTA \\
UTX & ATCCCAGCTCAGCAGAAGTT & GTGAGGATGGTGGTCTTGGA \\
$\beta$-actin & CCACTGGCATCGTGATGGACTCC & GCCGTGGTGGTGAAGCTGTAGC \\
\hline
\end{tabular}



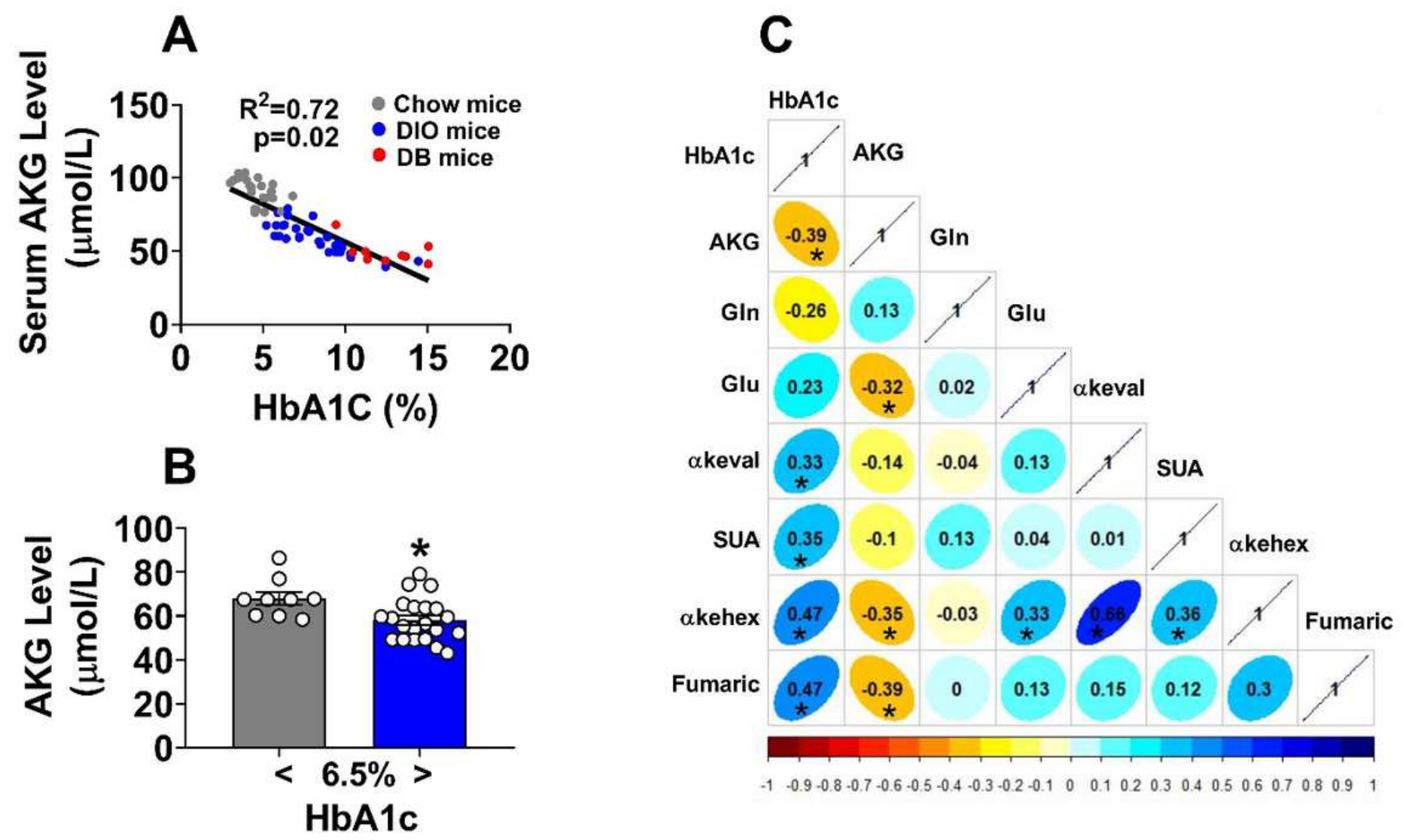

Figure 1

Serum AKG level is negatively with HbA1c. (A). Two-tailed Pearson's correlation coefficient analysis of plasma AKG level and $\mathrm{HbA1c}$ in mice. Chow male mice were fed a chow diet at 8 weeks of age $(n=21)$. For diet induced obesity mice (DIO), eight-week-old C57BL/ 6 male mice were fed HFD for 12 weeks ( $\mathrm{n}=$ $30)$. DB ( $d b / d b$ diabetes) male mice were fed a chow diet at 10 weeks of age $(n=9)$. (B). Plasma AKG level and HbA1c in DIO mice. Eight-week-old C57BL/6 male mice were fed HFD for 12 weeks ( $\mathrm{n}=9-21$ per group). (C). Two-tailed Pearson's correlation coefficient analysis of plasma AKG and related metabolite level with blood glucose in Chinese adults (36 males and 6 females). (Gln: glutamine; Glu: glutamic acid; a-keval: alpha-ketoisovaleric acid; SUA: succinic acid; a-kehex: a-ketoleucine; FUMA: fumaric acid; AKG: oxoglutaric acid). Data information: Results are presented as mean \pm SEM. In (B), $\unrhd p \leq 0.05$ by non-paired Student's t-test. 
A

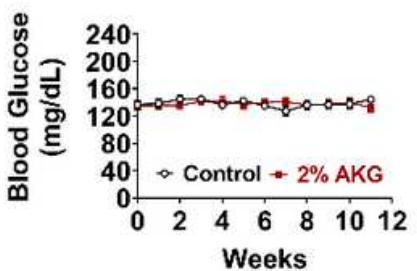

E

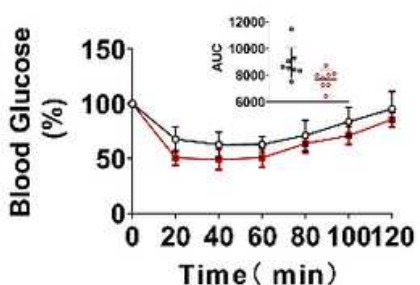

Time ( $\mathrm{min}$ )

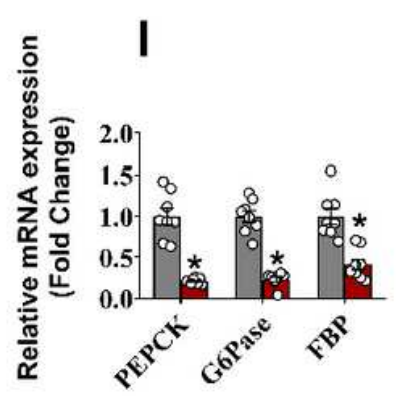

B

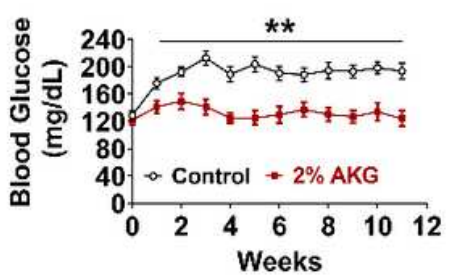

F

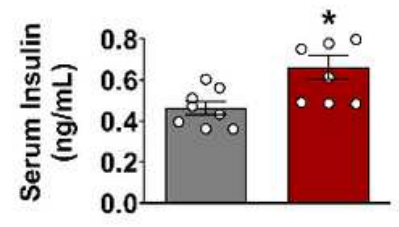

J

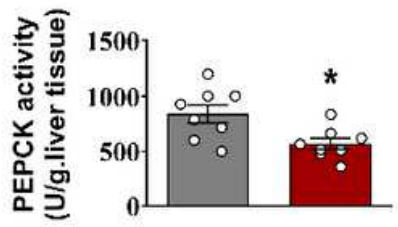

C

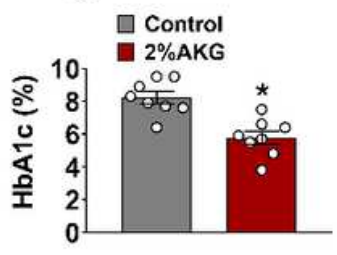

G

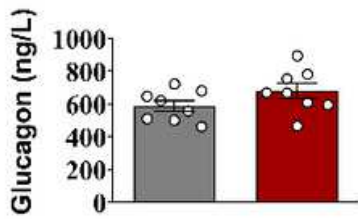

K

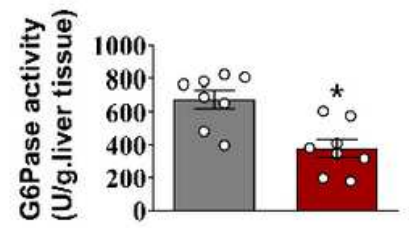

D

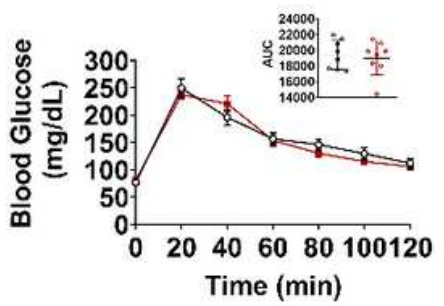

H
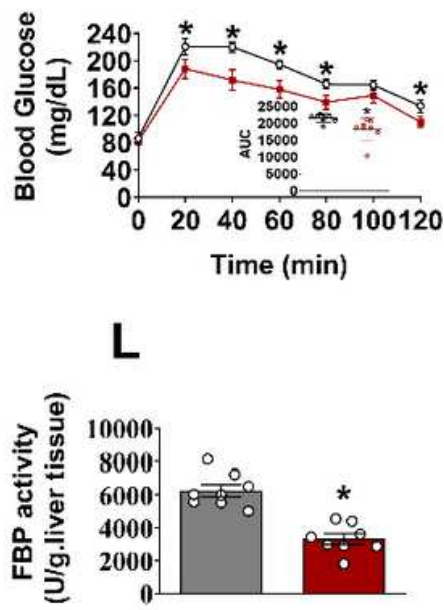

Figure 2

Chronic AKG supplementation prevents diet-induced hyperglycemia. (A). Blood glucose of male C57BL/6 mice. At 8 weeks of age, mice were fed a chow diet and received tap water or water supplemented with $2 \%$ AKG for 11 weeks ( $n=8$ per group). (B-C). Blood glucose (B) and serum HbA1c level (C) of male C57BL/ 6 mice. At 12 weeks of age, mice were switched to HFD and received tap water or water supplemented with $2 \%$ AKG for 11 weeks ( $n=8$ per group). (D-E). Glucose tolerance test $(1 \mathrm{~g} / \mathrm{kg}$ ) (D) and insulin tolerance test $(1 \mathrm{U} / \mathrm{kg})(\mathrm{E})$ in male C57BL/ 6 mice. At 12 weeks of age, mice were switched to HFD and received tap water or water supplemented with $2 \%$ AKG for 11 weeks ( $n=8$ per group). (F-G). Serum insulin level (F) and glucagon level (G) in male C57BL/6 mice. At 12 weeks of age, mice were switched to HFD and received tap water or water supplemented with $2 \%$ AKG for 11 weeks ( $n=8$ per group). (H). Pyruvate tolerance test (PTT, $1 \mathrm{~g} / \mathrm{kg}$ ). At 12 weeks of age, mice were switched to HFD and received tap water or water supplemented with $2 \%$ AKG for 11 weeks ( $n=8$ per group). (I). mRNA expression of gluconeogenesis genes in the liver of male C57BL/ 6 mice. At 12 weeks of age, mice were switched to HFD and received tap water or water supplemented with $2 \%$ AKG for 11 weeks $(n=8$ per group). (J-L). The activity of PEPCK $(\mathrm{J})$, G6Pase $(\mathrm{K})$, and FBP $(\mathrm{L})$ in liver of male C57BL/6 mice. At 12 weeks of age, mice were switched to HFD and received tap water or water supplemented with $2 \%$ AKG for 11 weeks ( $n=$ 8 per group). Data information: Results are presented as mean $\pm S E M$. In (B), (H). 『p $\leq 0.05$ by two-way ANOVA followed by post-hoc Bonferroni tests. In (C), $(\mathrm{F})$, and $(\mathrm{J}-\mathrm{L}),{ }^{*} \mathrm{p} \leq 0.05$ by non-paired Student's t-test. 

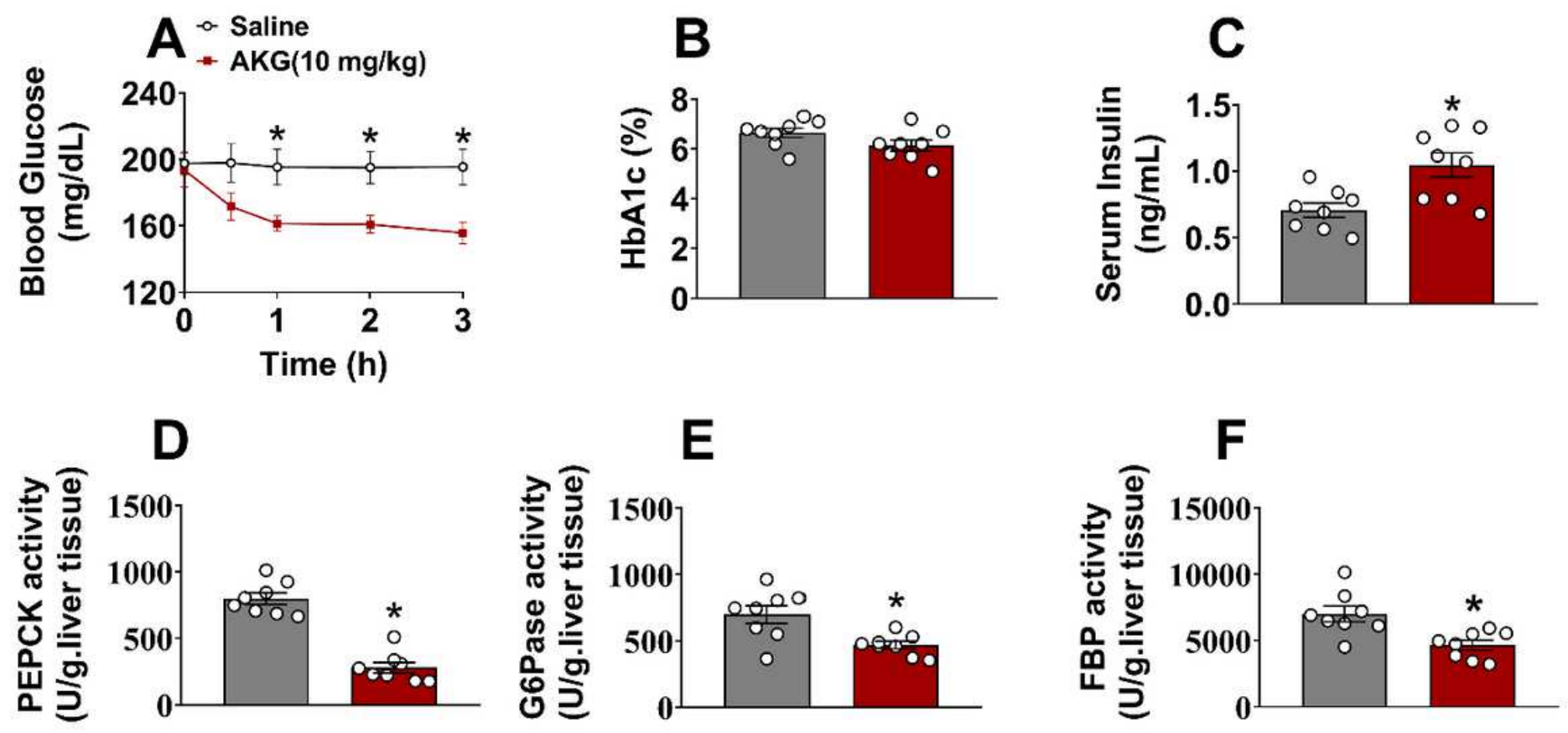

Figure 3

Acute AKG administration prevents diet-induced hyperglycemia. (A). Blood glucose concentration-time profile obtained from male C57BL/6 mice (10 weeks old) fed with HFD i.p saline or AKG $(10 \mathrm{mg} / \mathrm{kg}$ body weight). The blood glucose was tested at $0,0.5,1,2$, and 3 hrs after injection ( $\mathrm{n}=8$ per group). (B-C). Serum HbA1c level (B) and insulin level (C) in male C57BL/6 mice (10 weeks) fed with HFD i.p saline or AKG (10 mg/kg) for 3 hrs. (D-F). The activity of PEPCK (D), G6Pase (E), and FBP (F) in liver of male C57BL/6 mice (10 weeks) fed with HFD i.p saline or AKG $(10 \mathrm{mg} / \mathrm{kg})$ for $3 \mathrm{hrs}$. Data information: Results are presented as mean \pm SEM. In (A), $\Downarrow p \leq 0.05$ by two-way ANOVA followed by post-hoc Bonferroni tests. In (C-F), ${ }^{*} \mathrm{p} \leq 0.05$ by non-paired Student's t-test. 


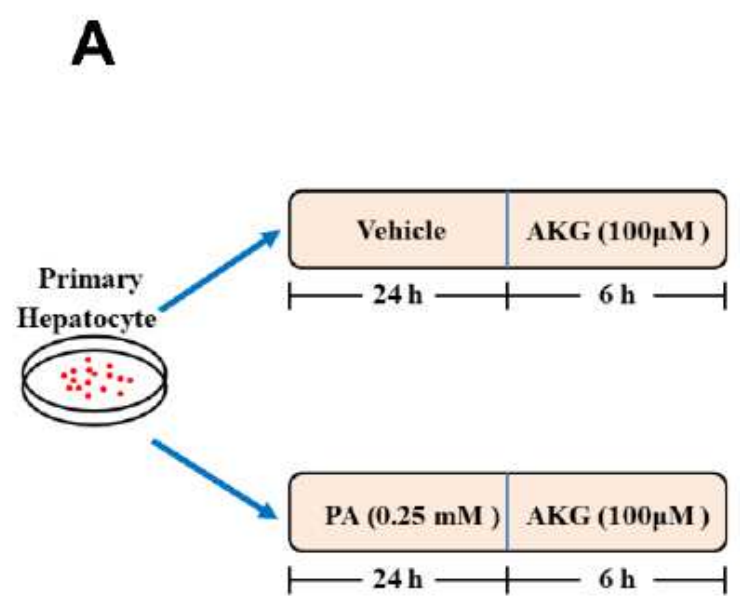

H

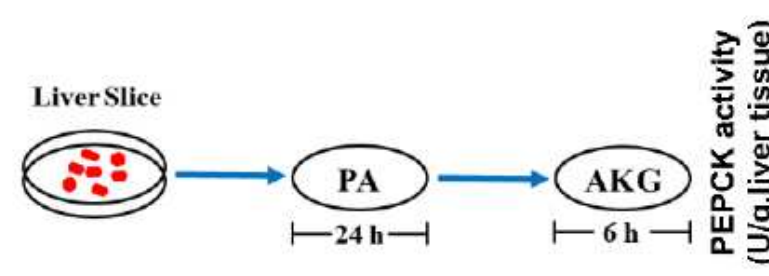

$\mathbf{L}$

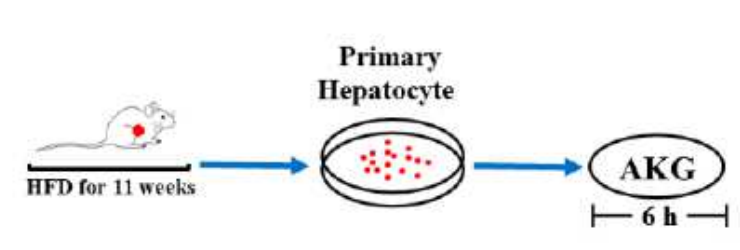

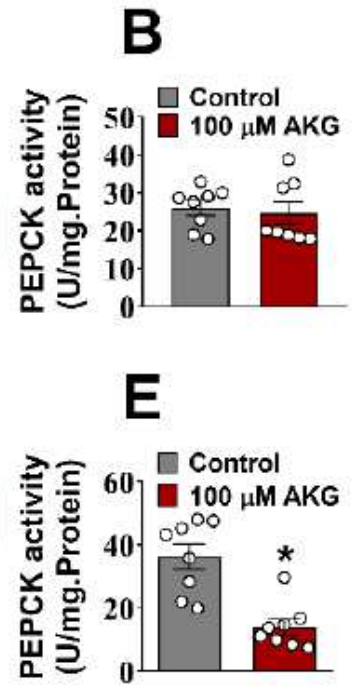

B

I
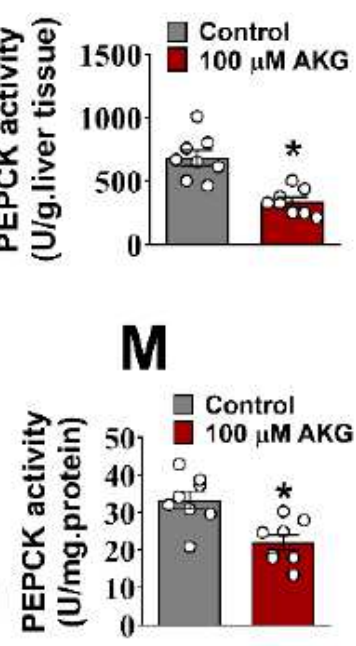

C

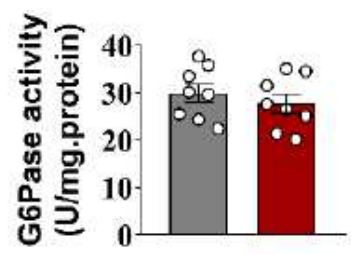

$\mathbf{F}$

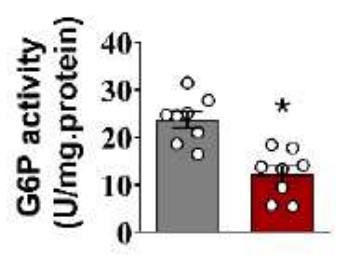

J

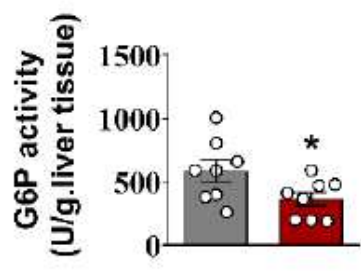

$\mathbf{N}$

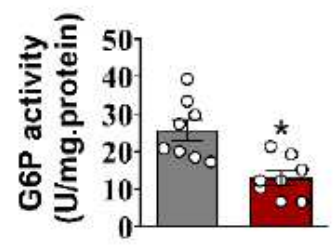

D

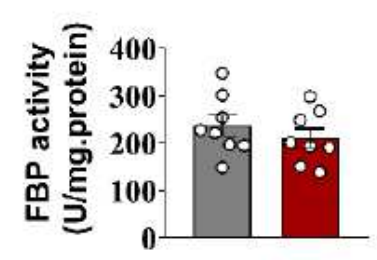

\section{G}
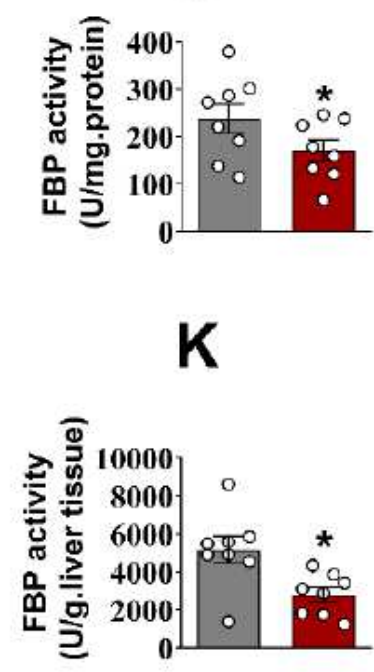

O

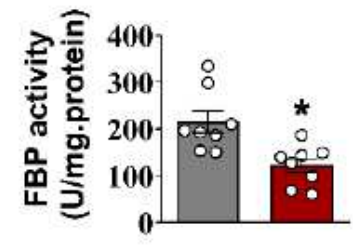

\section{Figure 4}

AKG suppresses hepatic gluconeogenesis in vitro. (A). Schematic representation of primary hepatocyte treated with AKG. 10 weeks of male C57BL/ 6 mice primary hepatocyte were cultured with vehicle or 0.25 $\mathrm{mM}$ PA for $24 \mathrm{~h}$, then treated with vehicle or $100 \mu \mathrm{M}$ AKG for $6 \mathrm{hrs}(\mathrm{n}=8$ per group). (B-D). The activity of PEPCK (B), G6Pase (C), and FBP (D) of primary hepatocyte. Primary hepatocytes were treated with vehicle or $100 \mu M$ AKG for 6 hrs ( $n=8$ per group). (E-G). The activity of PEPCK (E), G6Pase (F), and FBP (G) of primary hepatocyte. Primary hepatocyte were cultured with vehicle or $0.25 \mathrm{mM}$ PA for $24 \mathrm{hrs}$ and then treated with vehicle or $100 \mu M$ AKG for 6 hrs ( $n=8$ per group). $(H)$. Schematic representation of liver slice treated with AKG. C57BL/ 6 male mice were fed a chow diet at 10 weeks of age. Liver slices were cultured with $0.25 \mathrm{mM} \mathrm{PA}$ for $24 \mathrm{hrs}$ and then treated with vehicle or $100 \mu \mathrm{M}$ AKG for $6 \mathrm{hrs}(\mathrm{n}=8$ per group). (I-K). The activity of PEPCK (I), G6Pase (J), and FBP (K) of liver ( $\mathrm{n}=8$ per group). 


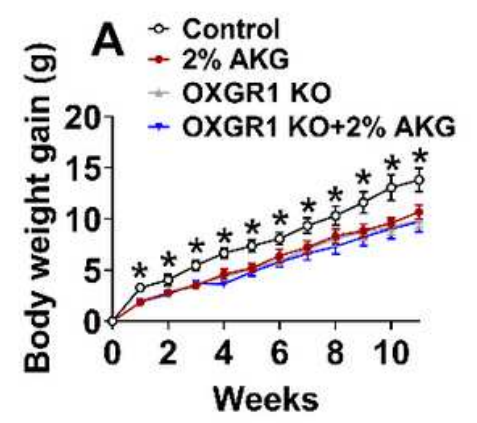

$\mathbf{E}$

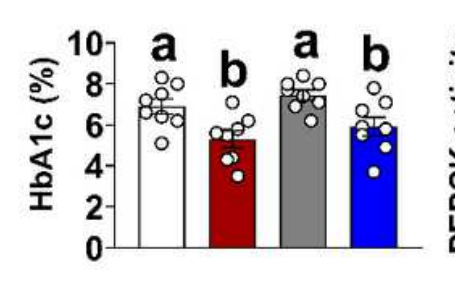

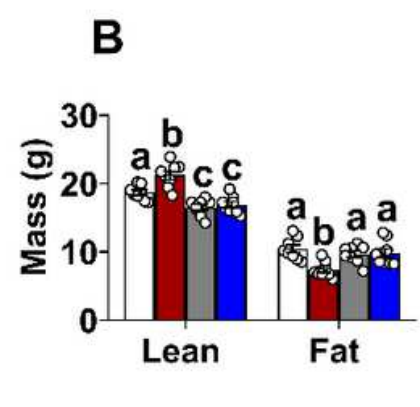

$\mathbf{F}$

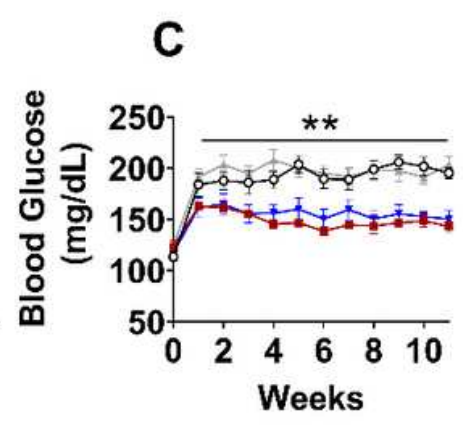

G

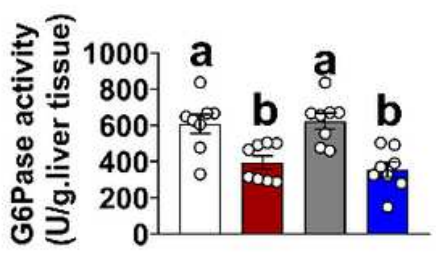

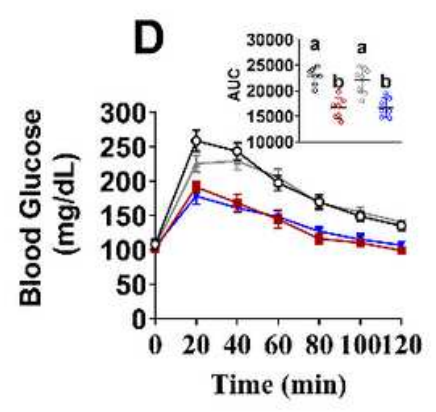

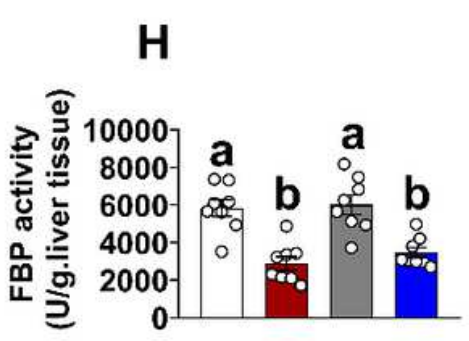

Figure 5

OXGR1 is not required for AKG- induced gluconeogenesis suppression. (A-C). Body weight gain (A), body composition (B), and blood glucose (C) of male WT control (littermates) or OXGR1KO mice. At 12 weeks of age, both control and KO mice were switched to HFD and further divided into two groups, receiving tap water or water supplemented with $2 \%$ AKG for 11 weeks ( $n=8$ per group). (D). Pyruvate tolerance test (PTT, $1 \mathrm{~g} / \mathrm{kg}$ ). At 12 weeks of age, both control and KO mice were switched to HFD and further divided into two groups, receiving tap water or water supplemented with $2 \%$ AKG for 11 weeks ( $n=8$ per group). (E). Serum HbA1c level. At 12 weeks of age, both control and KO mice were switched to HFD and further divided into two groups, receiving tap water or water supplemented with $2 \%$ AKG for 11 weeks ( $n=8$ per group). (F-H). The activity of PEPCK (F), G6Pase (G), and FBP (H) in the liver. At 12 weeks of age, both control and KO mice were switched to HFD and further divided into two groups, receiving tap water or water supplemented with $2 \%$ AKG for 11 weeks ( $n=8$ per group). Data information: Results are presented as mean \pm SEM. In (A, C), *p $\leq 0.05$ by two-way ANOVA followed by post-hoc Bonferroni tests. In (D-H), different letters between bars indicate $p \leq 0.05$ by one-way ANOVA followed by post-hoc Tukey's tests. 
A
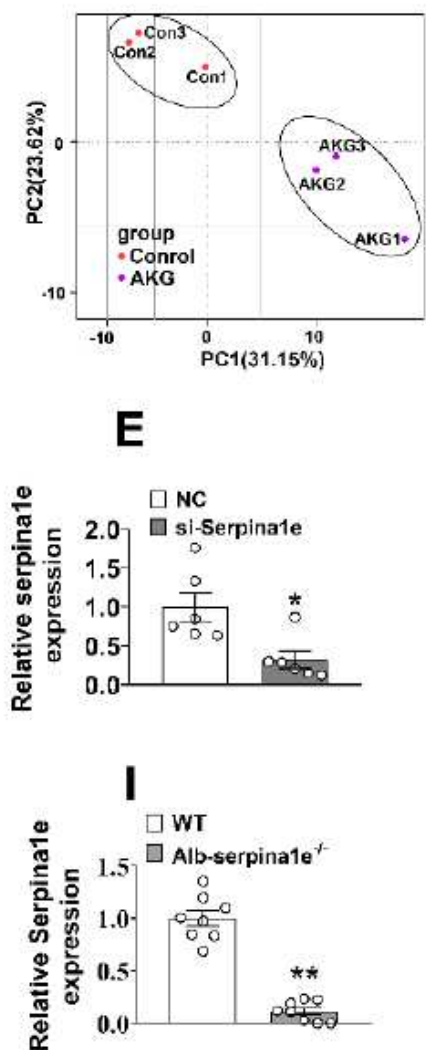

M

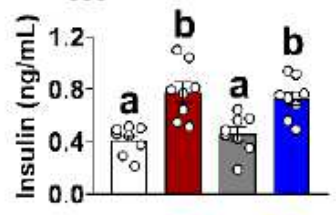

B
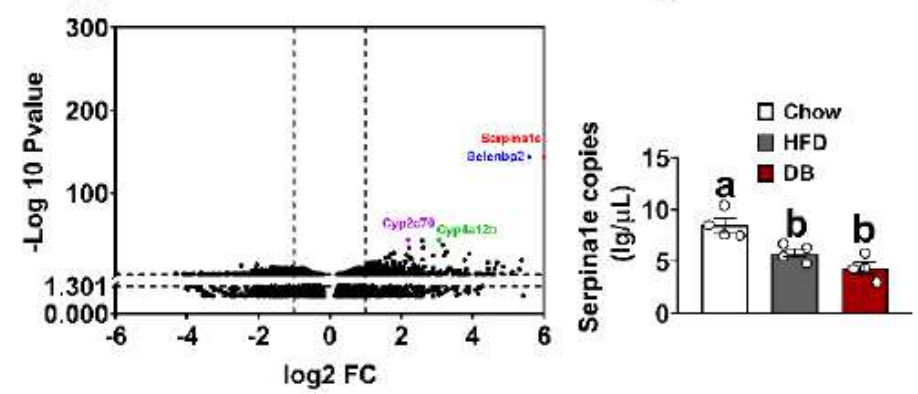

G
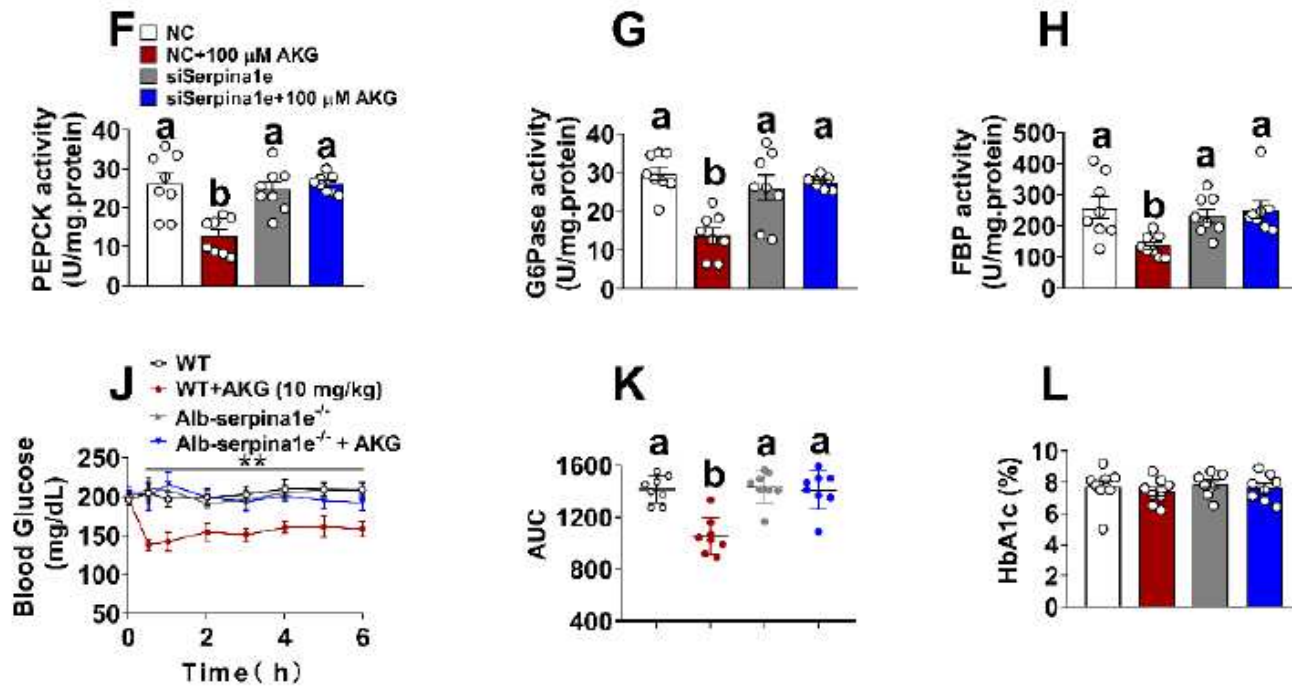

\section{N}

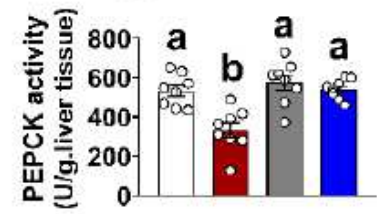

O

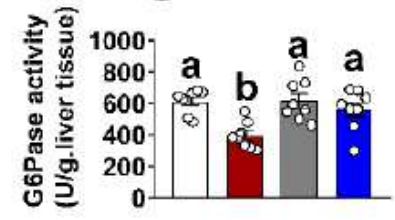

$\mathbf{L}$

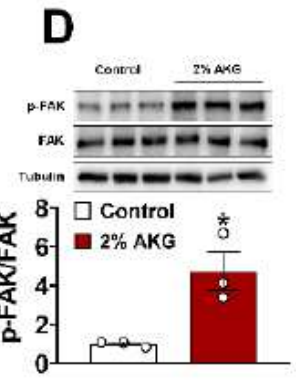

H

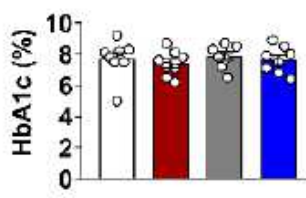

\section{Figure 6}

Serpina1e is required for the inhibitory effects of AKG on hepatic gluconeogenesis. (A). Principal coordinate analysis plot ( $n=3$ per group). (B). Volcano plot of AKG-induced transcriptome signature. Genes with $\log 2 \mathrm{FC} \geq 1$ and $-\log 10 \mathrm{P}$ value $\geq 1.3$ were considered significant. Serpina1e (red dots), selenbp2 (blue dots), cyp2c70 (purple dots), and cyp4a12b (green dots) genes were most significantly different between groups. At 12 weeks of age, mice were switched to HFD and received tap water or water supplemented with $2 \%$ AKG for 11 weeks ( $n=3$ per group). (C). mRNA expression of serpina 1 e in the liver tissue. Eight-week-old C57BL/6 male mice were divided into two groups and then fed a chow diet or HFD for 12 weeks. Eight-week-old db/db mice (DB) were fed a chow diet for 12 weeks ( $n=4$ per group). (D). Immunoblots and quantification of p-FAK protein expression in liver. At 12 weeks of age, mice were switched to HFD and received tap water or water supplemented with $2 \%$ AKG for 11 weeks ( $n=3$ per group). (E). mRNA expression of serpina1e in primary hepatocyte. Primary hepatocyte were transfected with negative control (NC) siRNA or si-serpina1e for $24 \mathrm{hrs}$ ( $\mathrm{n}=6$ per group). ( $\mathrm{F}-\mathrm{H})$. The activity of PEPCK $(\mathrm{F})$, G6Pase $(\mathrm{G})$, and FBP $(\mathrm{H})$ in primary hepatocyte (PA treatment) cultured with vehicle $+\mathrm{NC}$, vehicle + si- 
serpina1e, AKG $(100 \mu M)+N C$, or AKG + si-serpina1e for $6 \mathrm{~h}$ ( $\mathrm{n}=8$ per group). (I). mRNA expression of serpina1e in liver ( $\mathrm{n}=8$ per group). Alb-Cre mice were crossed with LSL-Cas9-EGFP mice to generate AlbCre/LSL-Cas9-EGFP (Alb-Cas9), a mouse model with Cas9 selectively overexpressed in Alb positive liver cells. Six-week-old male Alb-Cas9 mice were i.p injected with AAV-sgRNAs-serpina1e $(1 \times 1012 \mathrm{GC} / \mathrm{ml})$ to generate a liver-specific sepina1e deletion mouse model (Alb-serpina1 e-/-). (J-K). Blood glucose concentration-time profile (J-K) obtained from male WT control (littermates) or Alb-serpina1e-/- mice (10 weeks) fed with HFD i.p saline or AKG $(10 \mathrm{mg} / \mathrm{kg}$ body weight). The blood glucose was tested at 0 , $0.5,1,2,3,4,5$, and 6 hrs after injection ( $n=8$ per group). ( $L-M)$. Serum HbA1c level $(L)$ and insulin level (M) in male WT control (littermates) or Alb-serpina1e-/- mice (10 weeks) fed with HFD i.p saline or AKG (10 mg/kg body weight) at $0,0.5,1,2,3,4,5$, and $6 \mathrm{hrs}$ after injection ( $=8$ per group). (N-P). The activity of PEPCK $(\mathrm{N})$, G6Pase (O), and FBP (P) in the liver of male WT control (littermates) or Alb-serpina1e-/mice (10 weeks) fed with HFD i.p saline or AKG (10 mg/kg body weight) at 0, 0.5, 1, 2, 3, 4, 5, and $6 \mathrm{hrs}$ after injection ( $n=8$ per group). Data information: Results are presented as mean \pm SEM. In (C), $(F-H),(K)$, and $(M-P)$, different letters between bars indicate $p \leq 0.05$ by one-way ANOVA followed by post-hoc Tukey's tests. In (D-E) and (I-J), * $\mathrm{p} \leq 0.05$ by non-paired Student's t-test.
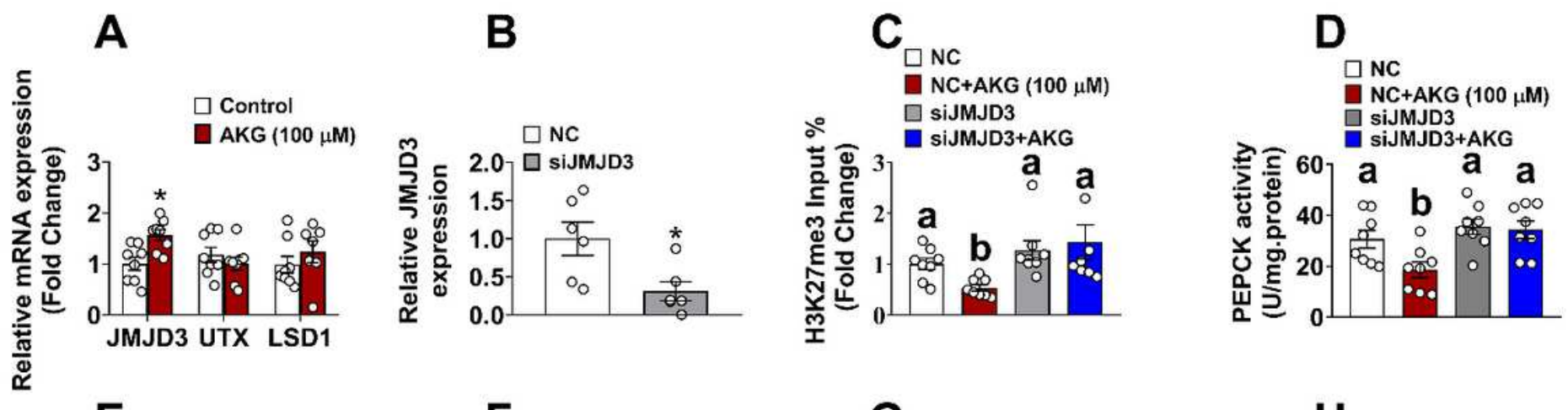

\section{$\mathbf{E}$}

$\mathbf{F}$
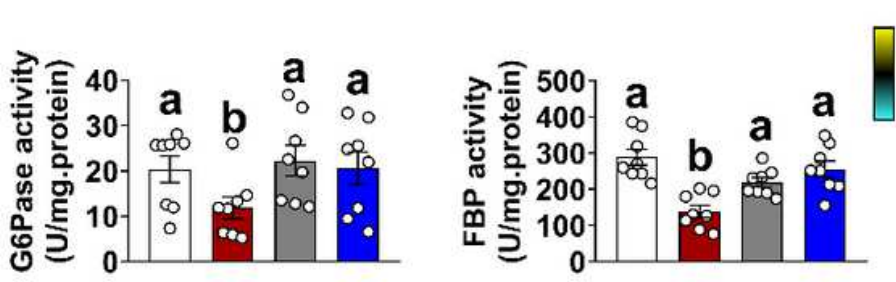

G

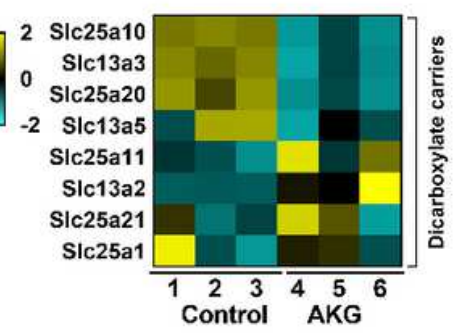

H
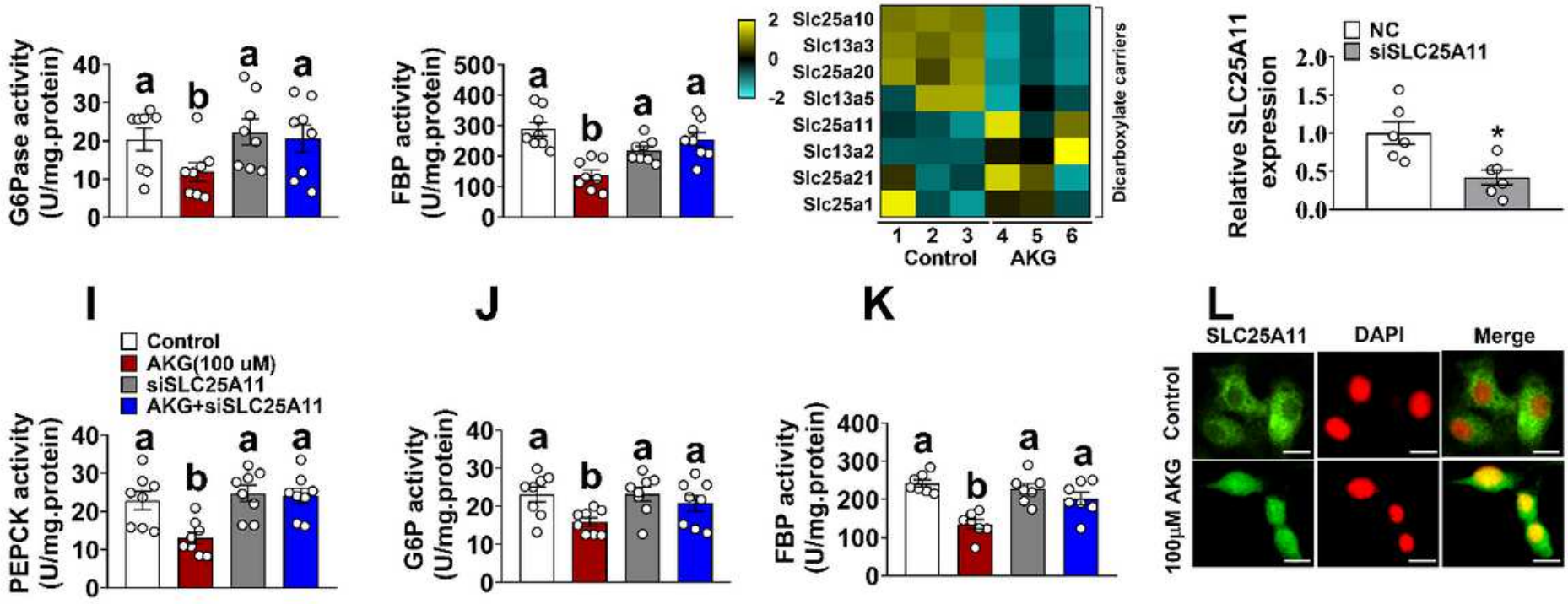

Figure 7 
Serpina1e mediates the inhibitory effects of AKG on hepatic gluconeogenesis. (A). mRNA expression of JMJD3, LSD, and UTX in primary hepatocyte. Primary hepatocytes were cultured with $0.25 \mathrm{mM}$ PA for 24 hrs and then treated with vehicle or $100 \mu \mathrm{M}$ AKG for 6 hrs ( $n=8$ per group). (B). mRNA expression of JMJD3 in primary hepatocyte. Primary hepatocytes were transfected with NC siRNA or si-SLC25A11 for 24 hrs ( $n=6$ per group). (C). Chromatin-immunoprecipitation (ChIP) analysis of H3K27me3 in promoter of serpina1e in primary hepatocyte (PA treatment) cultured with vehicle $+\mathrm{NC}$, vehicle + si-JMJD3, AKG $(100 \mu M)+N C$, or AKG + si-JMJD3 for 6 hrs $(n=8$ per group). (D-F). The activity of PEPCK (D), G6Pase $(E)$, and FBP $(F)$ in primary hepatocyte (PA treatment) cultured with vehicle + NC, vehicle + si-JMJD3, AKG $(100 \mu \mathrm{M})+\mathrm{NC}$, or AKG + si-JMJD3 for $6 \mathrm{hrs}$ ( $\mathrm{n}=8$ per group). (G). Relative changes of dicarboxylate carriers in response to AKG treatment. Heat maps show changes of dicarboxylate carriers in the mice liver. At 12 weeks of age, mice were switched to HFD and received tap water or water supplemented with $2 \%$ AKG for 11 weeks ( $n=3$ per group). $(H)$. mRNA expression of SLC25A11 in primary hepatocyte. Primary hepatocytes were treated with NC siRNA or si-serpina1e ( $n=6$ per group). (I-K). The activity of PEPCK $(\mathrm{I})$, G6Pase $(\mathrm{J})$, and FBP $(\mathrm{K})$ in primary hepatocyte (PA treatment) cultured with vehicle $+\mathrm{NC}$, vehicle + si-SCL25A11, AKG $(100 \mu M)+N C$, or AKG + si-SLC25A11 for 6 hrs ( $n=8$ per group). (L). Immunofluorescence of SLC25A11 translocation in primary hepatocyte. Primary hepatocytes were cultured with $0.25 \mathrm{mM} \mathrm{PA}$ for $24 \mathrm{~h}$ and then treated with vehicle or $100 \mu \mathrm{M}$ AKG for $6 \mathrm{hrs}(\mathrm{n}=8$ per group). Scale bars, $50 \mu \mathrm{m}$. Data information: Results are presented as mean \pm SEM. In (A-B) and $(H){ }^{*} p$ $\leq 0.05$ by non-paired Student's t-test. In (C-F) and (I-K), different letters between bars indicate $p \leq 0.05$ by one-way ANOVA followed by post-hoc Tukey's tests. 